\title{
Research Paper: \\ Mediating Role of External Shame and Self-compassion in the Relationship Between Peer Victimization and Depression in Adolescents
}

\author{
Soheila Yaghoubi ${ }^{1}$ (), ${ }^{*}$ Behrooz Birashk $^{1}$ (), Asma Aghebati ${ }^{1}$ (), Ahmad Ashouri ${ }^{1}$ (1)
}

1. Department of Clinical Psychology, School of Behavioral Sciences and Mental Health (Tehran Institute of Psychiatry), Iran University of Medical Sciences, Tehran, Iran.

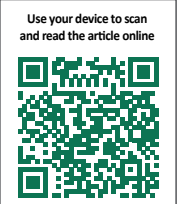

cittation Yaghoubi S, Birashk B, Aghebati A, Ashouri A. [Mediating Role of External Shame and Self-compassion in the Relationship Between Peer Victimization and Depression in Adolescents (Persian)]. Iranian Journal of Psychiatry and Clinical Psychology. 2021; 27(1):16-31. http://dx.doi.org/10.32598/ijpcp.27.1.3288.1

doi http://dx.doi.org/10.32598/ijpcp.27.1.3288.1

Received: 28 Dec 2019

Accepted: 02 Jun 2020 Available Online: 01 Apr 2021

Key words: Adolescents, Victimization, Shame, compassion, Depression

\section{A B STRACT}

Objectives The present study aims to examine the mediating role of external shame and self-compassion in the relationship between peer victimization and depression in adolescents.

Methods This is a descriptive-correlational study. Participants were 300 middle-school students of Shahin Shahr city in Isfahan, Iran (150 girls and 150 boys), who were selected using a multi-stage cluster sampling method. The data collection tools were Multidimensional Peer-Victimization Scale, the Other As Shamer Scale, Self-Compassionate Scale-Short Form, and Mood \& Feeling Questionnaire. The collected data were analyzed in SPSS v. 19 and AMOS v. 20 applications.

Results There were significant relationship among peer victimization, external shame, self-compassion, and depression $(\mathrm{P}<0.01)$. The measurement model had a good fitness (Root mean square error of approximation $=0.037$, normed fit index $=0.997$, comparative fit index $=0.999$ ). Path Analysis results reported that peer victimization had indirect effect on depression through mediation by external shame and self-compassion.

Conclusion External shame and self-compassion can mediate the relationship between peer victimization and depression in adolescents. Considering them in designing effective preventive and therapeutic interventions can be helpful for adolescents to cope with peer victimization and depression.

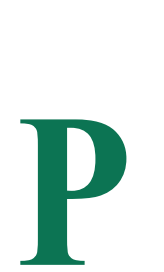

Extended Abstract

\section{Introduction}

eer victimization is the experience among children of being a target of the aggressive behavior of other children. Peer victimization is prevalent and can lead to mental health difficulties [4]. Among the outcomes of peer victimization, depression has been reported more; however, not all adolescents who experience victimization develop depression. Thus, identifying the mechanisms through which peer victimization is related to depression is important for development of interventions to reduce the negative impacts of peer victimization. Two potential mechanisms are external shame and self-compassion. External shame refers to how one perceives oneself as unattractive to others and, therefore, has a feelings of being rejected and vulnerable to attacks from others. It has a strong association with depression [19, 20]. Since adolescents are more sensitive to the images they create in others and being victimized by peers is an experience that involves humiliation, this phenomenon can make adolescents vulnerable to external shame and expose them to depression. However, studies have shown that shame has a mediating role which suggests that there are other psychological mechanisms. Another potential mechanism

\section{* Corresponding Author:}

Behrooz Birashk, PhD.

Address: Department of Clinical Psychology, School of Behavioral Sciences and Mental Health (Tehran Institute of Psychiatry), Iran University of Medical Sciences, Tehran, Iran.

Tel: +98 (31) 45247434

E-mail: birashk.b@iums.ac.ir 
Table 1. Correlation matrix of the study variables

\begin{tabular}{ccccc}
\hline Variables & Depression & Self-compassion & External Shame & Peer Victimization \\
\hline Peer victimization & $0.438^{* *}$ & $-0.328^{* *}$ & $0.551^{* *}$ & 1 \\
\hline External shame & $0.669^{* *}$ & $-0.433^{* *}$ & 1 & \\
\hline Self-compassion & $-0.566^{* *}$ & 1 & & \\
\hline Depression & 1 & & \\
\hline$* * \mathrm{P}<0.01$. & & $\begin{array}{c}\text { Iranian Journa of } \\
\text { PSYCHIATRY AND CUNICAL PSYCHOLOGY }\end{array}$
\end{tabular}

is self-compassion. A self-compassionate person has a realistic awareness of his/her strengths and weaknesses, sees them as part of the common experience in human beings and takes a balanced perspective on them [26, 27]. Peer victimization is associated with emotion dysregulation [32]. Thus, the development of self-compassion as an effective emotion regulation strategy can be impaired in victims. To our knowledge, no study on the role of self-compassion in the context of peer victimization has been conducted so far.

The mediating role of external shame and self-compassion can be well justified based on the compassionate mind theory of Gilbert. According to theory, humans are born with an innate desire to belong to a group and create positive emotions in others. Experiencing hostile environment can activate the threat system in which humans use safety strategies to protect themselves but these strategies may have unintended consequences such as rumination, feeling of unworthiness and depression. What sustain this cycle going is shame, self-attack and reduced self-compassion $[40,41]$. Based on this approach, the present study aims to examine the mediating role of external shame and selfcompassion in the relationship between peer victimization and depression.

\section{Methods}

This is a descriptive correlational study. The study population consists of all middle-school students in Shahin
Shahr city, Isfahan, Iran during 2018-2019. Samples were selected using a multi-stage cluster sampling method. The sample size was determined 300 considering a sample drop and given that there is need for at least 20 cases for each parameter [43]. To collect the data, Multidimensional PeerVictimization Scale, Other As Shamer Scale, Self-Compassionate Scale-Short Form, and Mood \& Feeling Questionnaire were used $[44,46,48,50]$. The data were analyzed in SPSS V. 19 and AMOS V. 20 applications.

\section{Results}

Pearson's correlation test results showed a significant correlation (Table 1). The results of path analysis showed that the direct paths between the study variables were significant except between peer victimization and depression; therefore, the path between them was removed (Figure 1). The evaluation of measurement model by using fit indices demonstrated that the measurement model had a good fitness (Table 2). To evaluate the mediating effect, the Bootstrap test was used. The results showed that all of the three indirect paths were significant (Table 3).

\section{Discussion and Conclusion}

The purpose of this study was to investigate the mediating roles of external shame and self-compassion in the relationship between peer victimization and depression. Findings showed that the study model had a good fitness. That is,

Table 2. Goodness-of-fit indices for the measurement model

\begin{tabular}{cccccccccc}
\hline Indices & X $2 / d f$ & PGFI & AGFI & GFI & RFI & CFI & TLI & NFI & RMSEA \\
\hline Results & 1.418 & 0.999 & 0.976 & 0.998 & 0.979 & 0.999 & 0.994 & 0.997 & 0.037 \\
Accepted range & $<2$ & $>0.95$ & $>0.95$ & $>0.95$ & $>0.95$ & $>0.95$ & $>0.90$ & $>0.95$ & $<0.05$ \\
\hline
\end{tabular}

Iranian Journal of
PSYCHIATRY AND CUINICAL PSYCHOLOGY $\mathrm{X}^{2}$ /df: Chi-square/degree of freedom; PGFI: Parsimony Goodness of Fit Index; AGFI: Adjusted Goodness of Fit Index, GFI: Goodness-of-Fit Index; RFI: Relative Fit Index; CFI: Comparative Fit Index; TLI: Tucker-Lewis Index; NFI: Normed Fit Index; RMSEA: Root Mean Square Error of Approximation. 
Table 3. Bootstrap test results for the mediating effect

\begin{tabular}{cccccc}
\hline \multirow{2}{*}{ Independent Variable } & Mediating Variable & Dependent Variable & \multicolumn{2}{c}{$95 \% \mathrm{Cl}$} & \multirow{2}{*}{ Effect Size } \\
\cline { 4 - 5 } & & & Lower Bound & Upper Bound & \\
\hline Peer victimization & External shame-Self-compassion & Depression & 0.011 & 0.583 & 0.399 \\
Peer victimization & External shame & Self-compassion & 0.030 & 0.742 & -0.199 \\
\hline External shame & Self-compassion & Depression & 0.045 & 0.650 & 0.123 \\
\hline
\end{tabular}

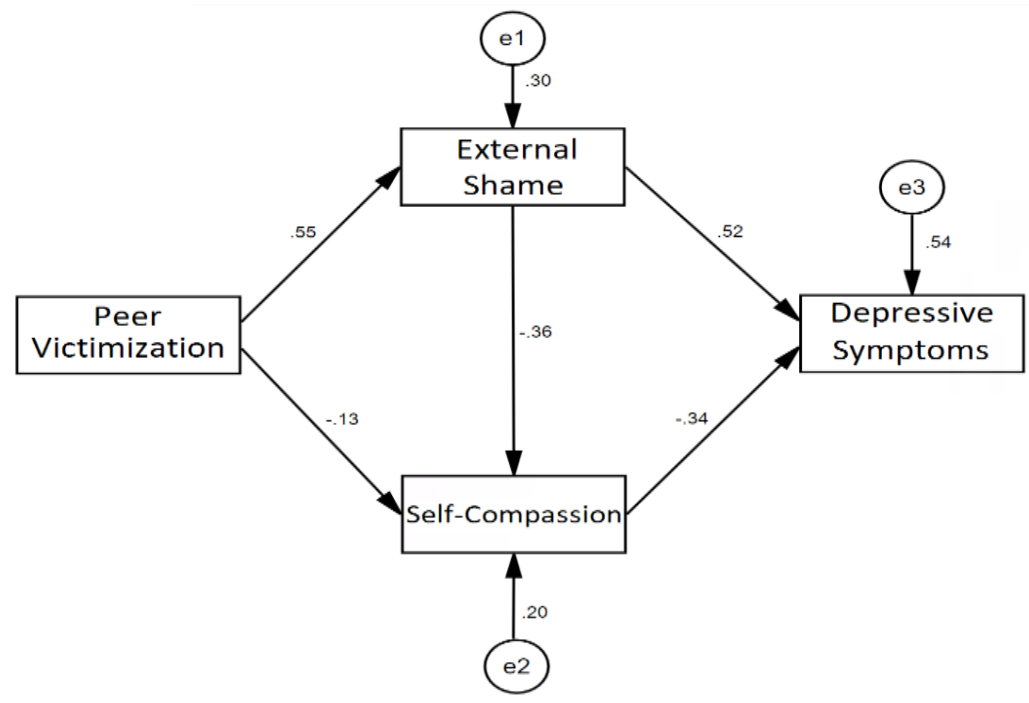

Figure1. Final study model with standardized path coefficients

students experiencing higher levels of peer victimization reported higher levels of external shame, which was associated with lower levels of self-compassion and depression. The results are consistent with the results of Irwin et al. Strom et al. Duarte et al. Jativa and Cerezo, and Zhang et al. [23-25, 35, 37]. Due to the increase in the sense of belonging and metacognitive abilities in adolescence and given that youth feel humiliated by peer victimization, the victim may think that others have a negative perceptions of him/ her. According to Gilbert, victimization by peers may create a hostile environment, stimulate the threat system and activate the fear of getting hurt by others [40]. External shame is associated with the secretion of cortisol (a steroid hormone related to the threat system) and accompanied with adopting coping strategies (avoidance, refusing to ask for help) which intensify depression. The victims may learn self-attack from a bully and lead to lack of self-compassion in them. People with low self-compassion are more likely to engage in selfcriticism [20] and judge their negative aspects which may be the same aspects targeted in peer victimization. Research
Iranian Journal of
PSYCHIATRY AND CUINICAL PSYCHOLOGY

has shown that self-criticism is one of important causes of depression, even years after becoming a victim.

These results of this study may help explain why peer victimization does not put anyone in a vulnerable position. In fact, peer victimization is associated with depressive symptoms, through believing in the negative judgment of others and consequently, through a kind and balanced attitude towards one's shortcomings and weaknesses. The importance of interventions that emphasize on reducing shame and increasing self-compassion in coping with peer victimization and treating depression can be the potential clinical implications of the results of this study.

\section{Ethical Considerations}

Compliance with ethical guidelines

This study has been approved by the ethics committee of Iran University of Medical Sciences (Code: IR. IUMS. REC.1398.099). According to research ethics, a written 
consent must be obtained from the parents, if the subjects are children (under 18 years of age). Also, one of the exclding criteria was the withdrawal of the participant from continuing the research. They were also assured about the confidentiality of their information and were free to leave the study whenever they wished, and if desired, the research results would be available to them.

\section{Funding}

This research did not receive any grant from funding agencies in the public, commercial, or non-profit sectors.

\section{Authors contributions}

Conceptualization: All authors; Methodology: Soheila Yaghoubi and Ahmad Ashouri; Date analysis: Soheila Yaghoubi and Ahmad Ashouri; Research: Asma Aqebati and Soheila Yaghoubi; writing - original draft: Behrouz Birshak and Soheila Yaghoubi; Writing - review \& editing: Asma Aqebati and Soheila Yaghoubi; Resources: Soheila Yaghoubi and Asma Aqbati; Supervision and coordination: Behrooz Birshak.

\section{Conflicts of interest}

The authors declare no conflict of interest.

\section{Acknowledgements}

The authors would like to thank the Education Organization of Isfahan and all participants for their cooperation. 
This Page Intentionally Left Blank 


\title{
مقاله بيزوهشى:
}

نقش ميانجى شرم بيرونى وشفقت به خود در رابطه بين قربانى شدن توسط همرسالان و نشانههاي

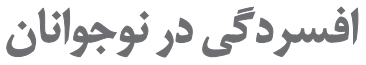

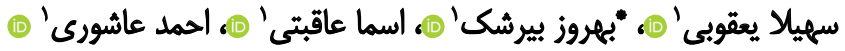

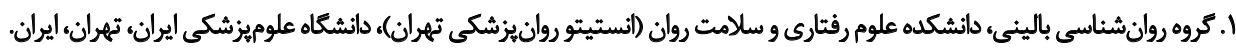

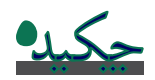

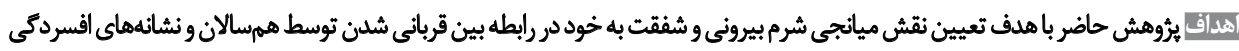

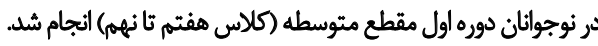

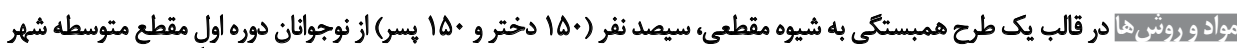

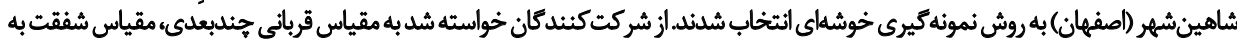

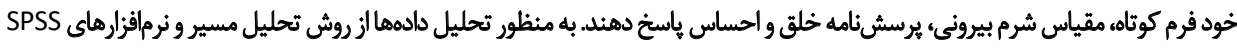

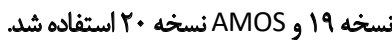

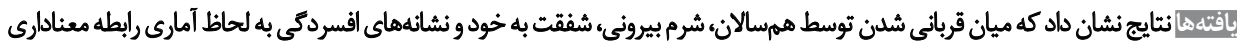

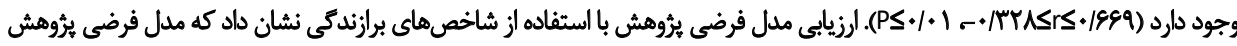

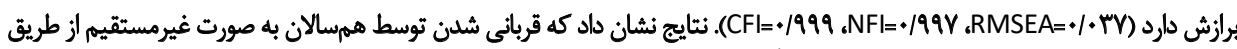

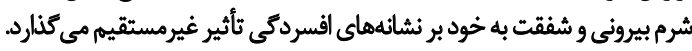

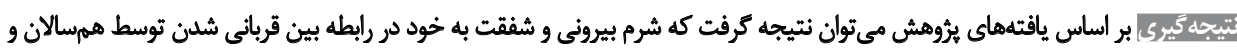

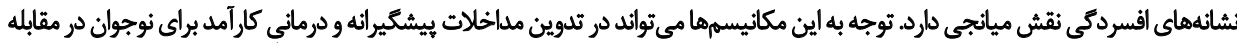

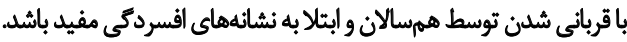

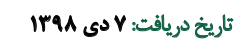

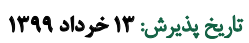

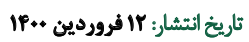

كليدوازوها:

قربانى شدن توسط

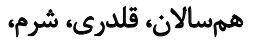

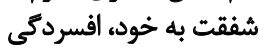

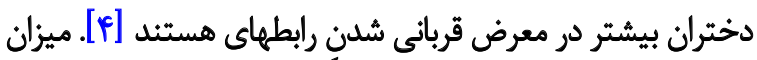
مقدمه

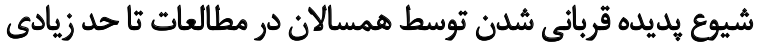

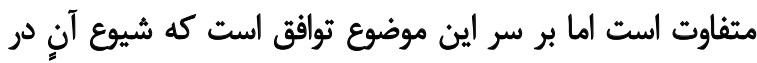

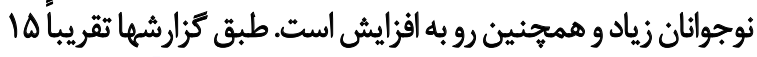

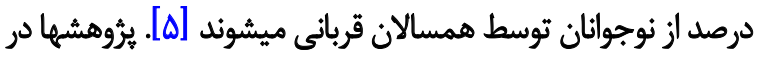

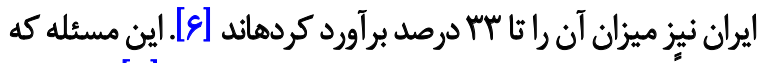

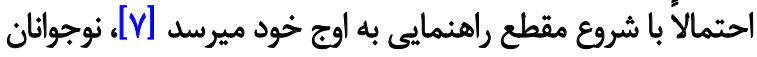

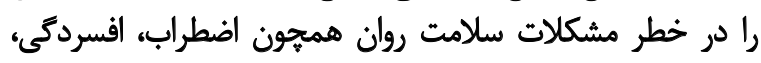

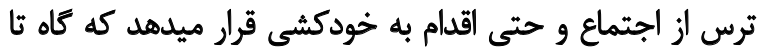

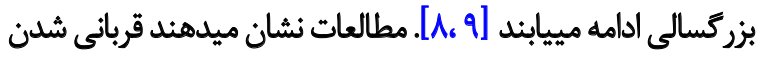

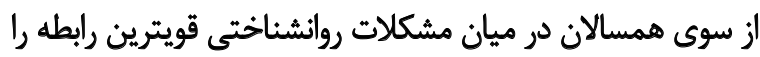

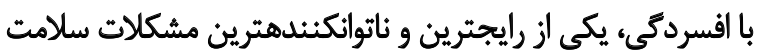

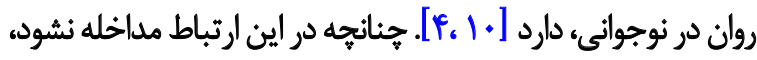

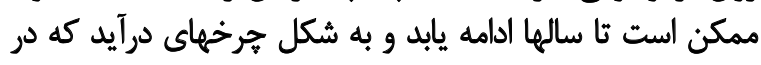

دوره انتقال بين كودكى و بزركسالى به خاطر ثلاقى تغييرات

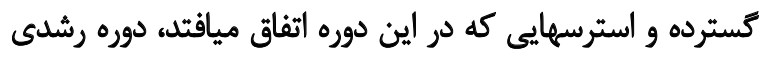

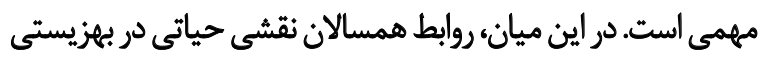

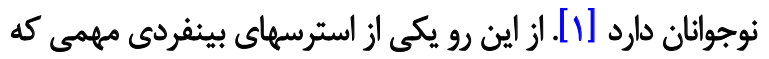

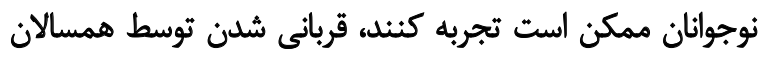

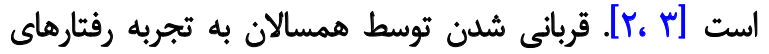

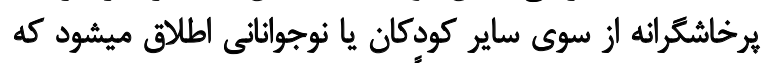

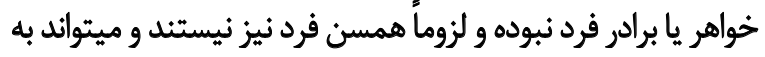

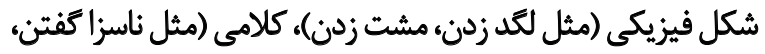

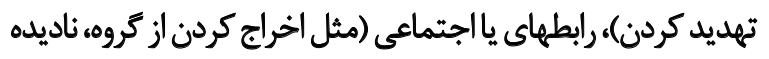

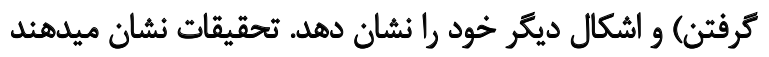

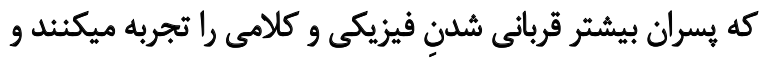


مي كثند حساس هستند و قربانى شدن توسط همرسالان تجربهاى

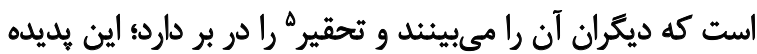

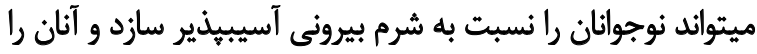

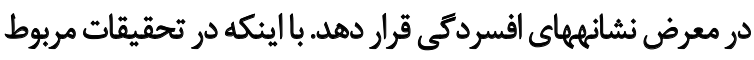

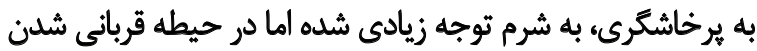

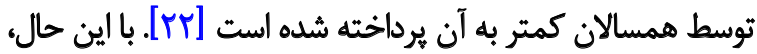

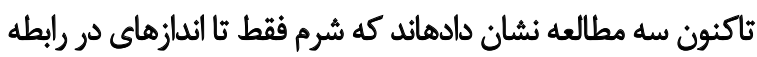
قربانى شدن توسط همسالان و نار احتى روانشناختى نقانش مياني

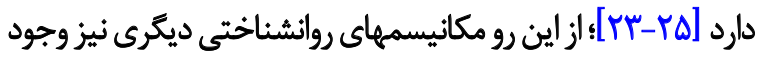

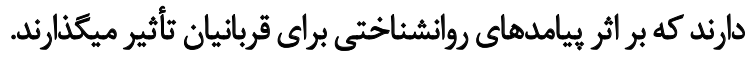
يك مكانيسم روانشناختى احتمالى ديخر كه رابطه بين قربانى

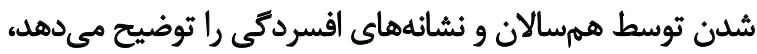

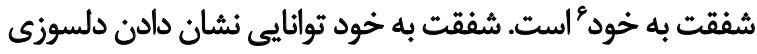

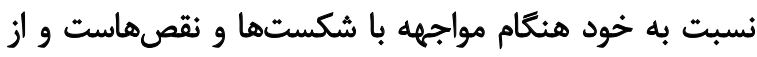

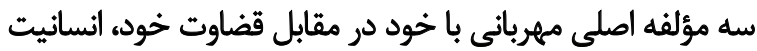

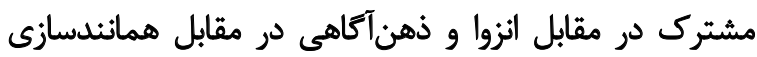

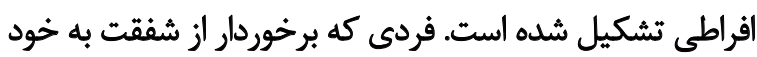

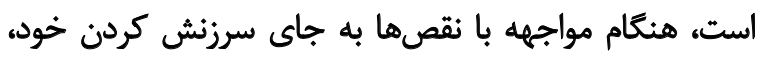

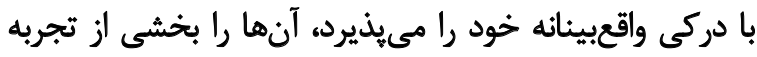

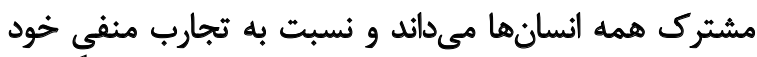

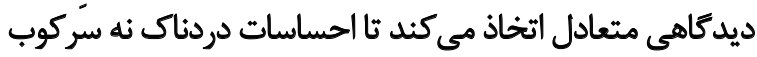

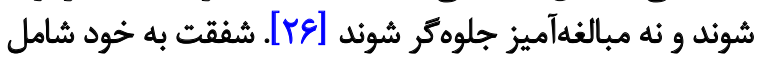

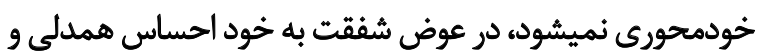

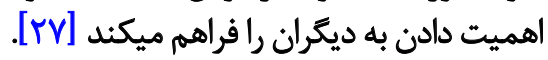

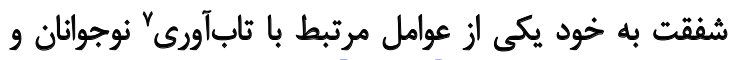

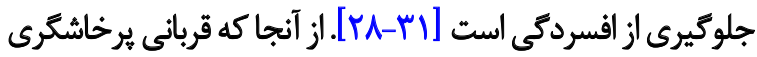

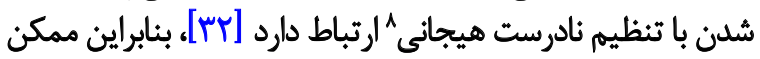

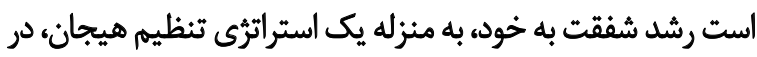

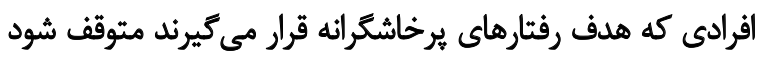

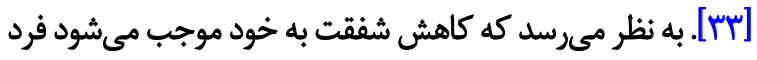

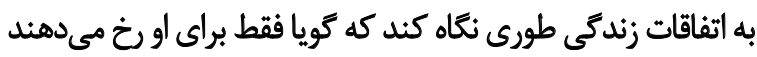

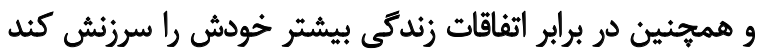

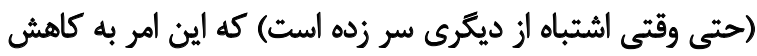

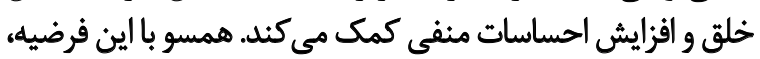

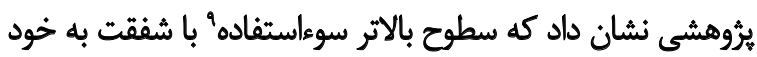

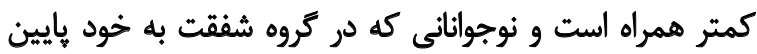

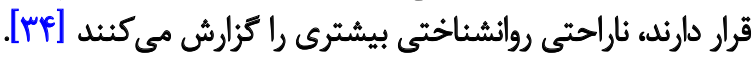

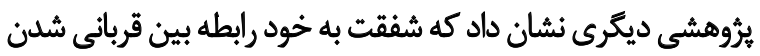

\section{Humiliation}

6. Self-Compassion

7. Resilience

8. Emotional dysregulation

9. Abuse

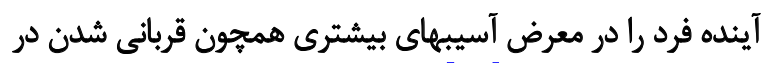

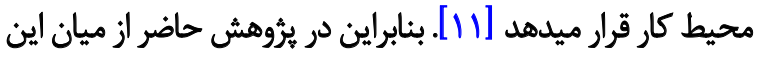
ييامدهاى منفى، به ارتباط قربانى شدن توسط همسالان و افسردئى

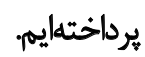

شرجند مطالعات مقطعى و طولى نشان ميدهند كه قربانى

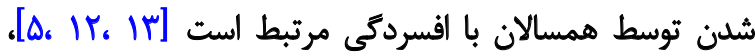

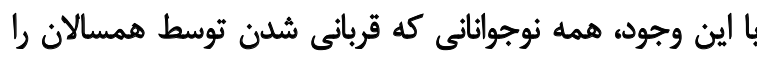

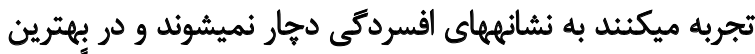

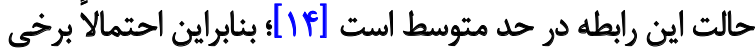

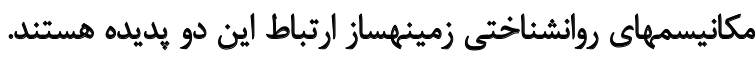

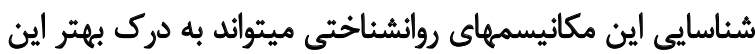

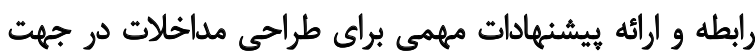

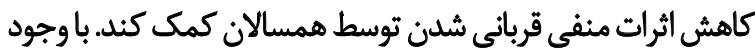

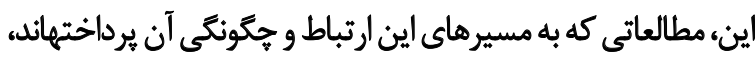

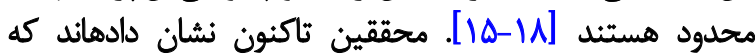

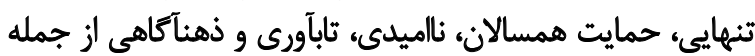

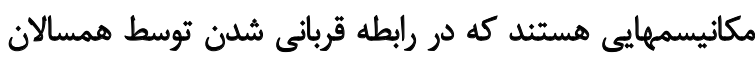

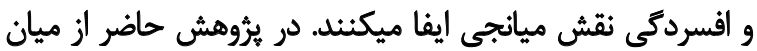

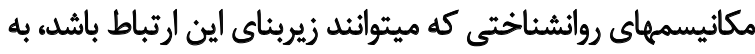
شرم بيرونى و شفقت به خود توجه كردهايم.

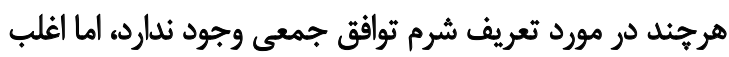

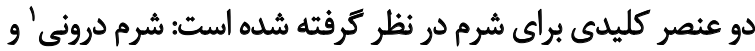

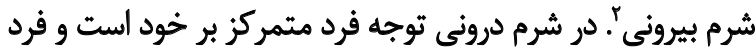

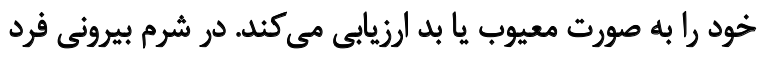

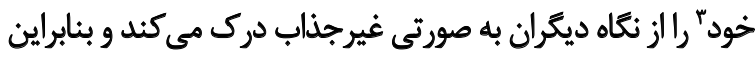

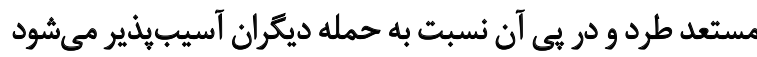

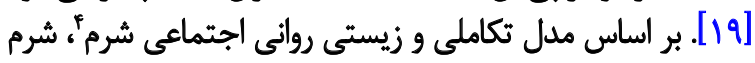

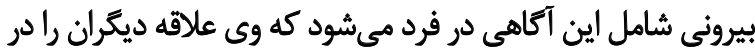

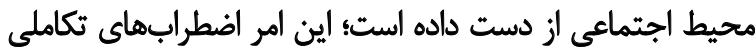

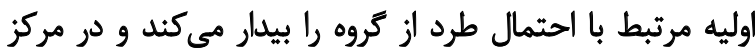

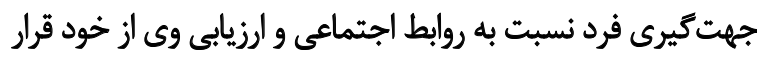

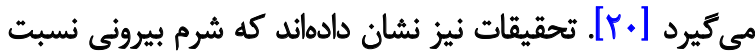

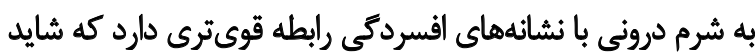

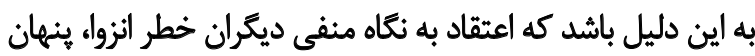

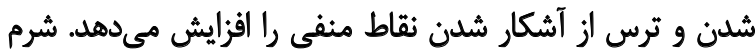

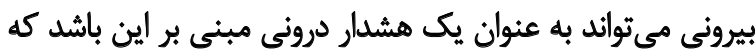

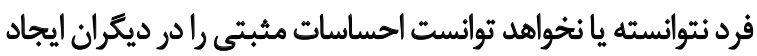

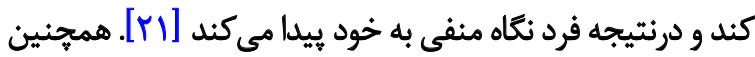
از آنجا كه نوجوانان نسبت به تصاوير و هيجانائى كه در ديكران ايجاد
1. Internal Shame
2. External Shame
3. Self
4. An evolutionary and biopsychosocial model for shame 
در همين راستا، قرباني شدن توسط همبسالان نيز مي تواند سيستم

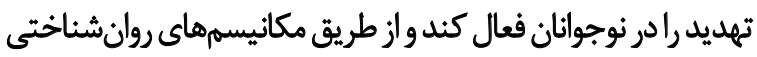

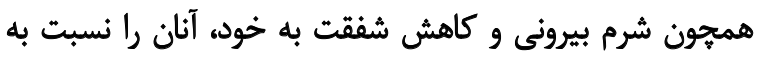

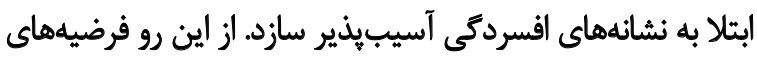

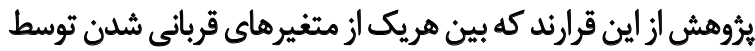

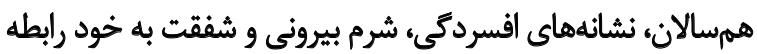

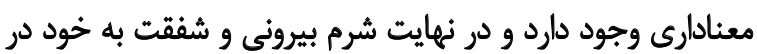
رابطه بين قربانى شدن و افسردگى نقش ميانجى دارند.

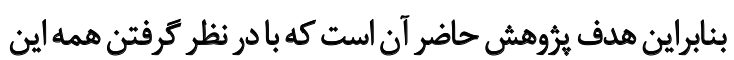

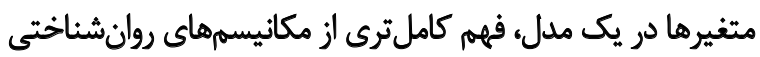

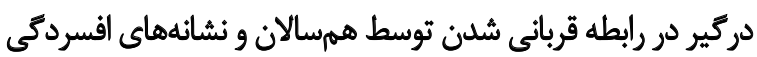

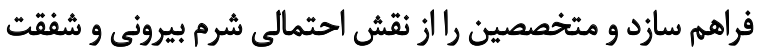

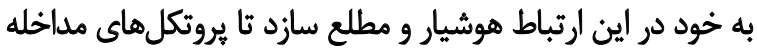

را بهبود بنخشيند.

ووش

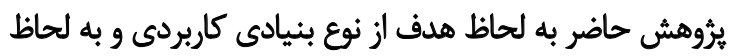

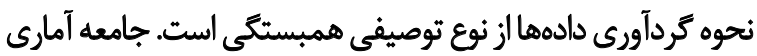

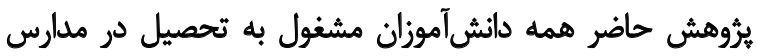

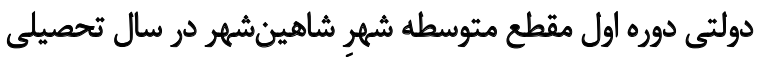

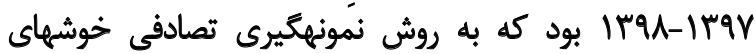

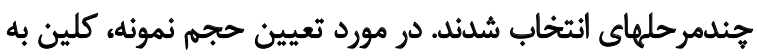

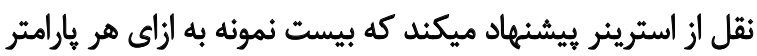

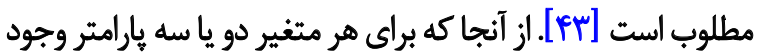

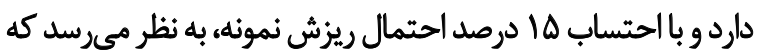
حجم نمونه سيصد نفر كفايت مي كند.

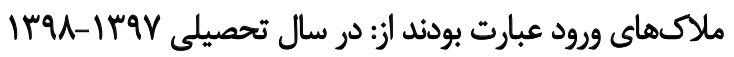

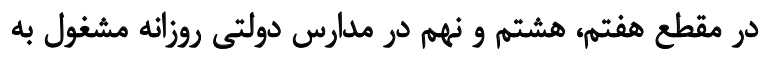

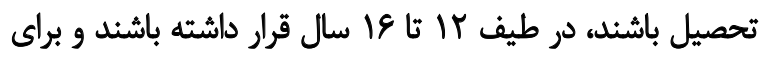

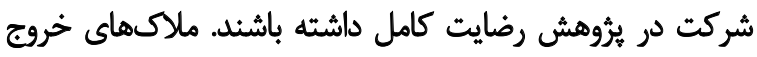

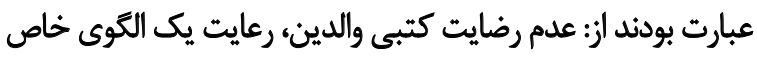

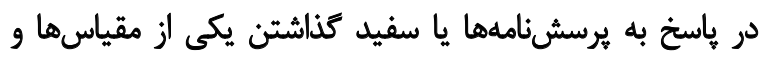

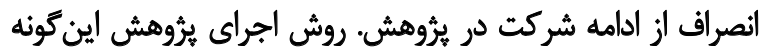

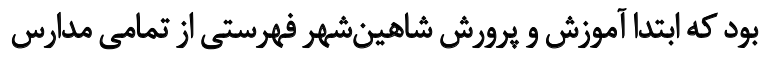

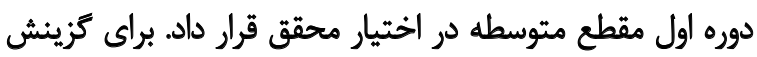

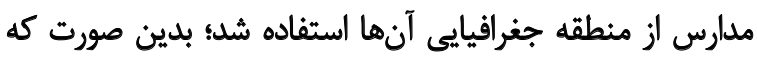

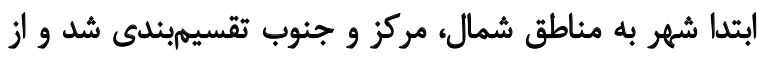

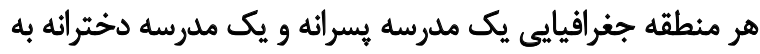

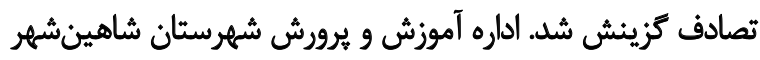

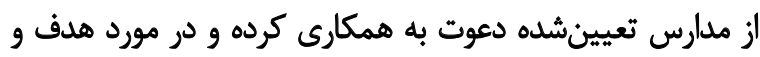

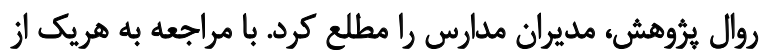

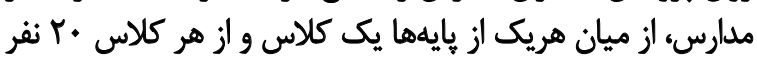
به صورت تصادفى انتخاب شد. با توجه به اينكه شركت كنيندكان
(به صورت كلى) و ناسازگكارى روانشناختى در نوجوانان را به طور

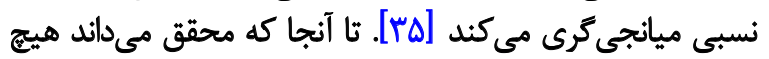

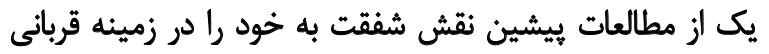

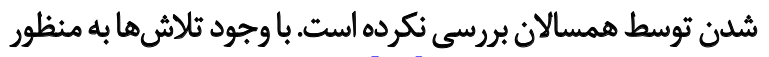

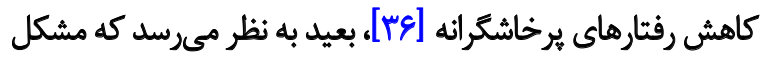

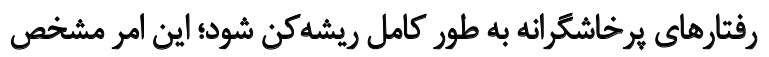

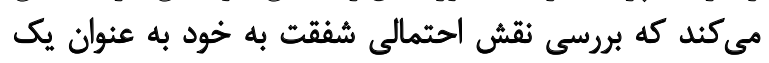

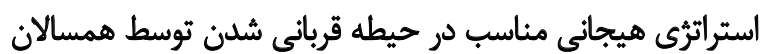
تا تا ميه ميزان لازم است.

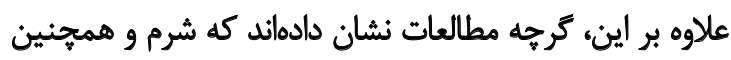

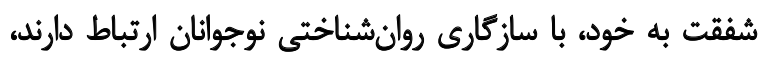

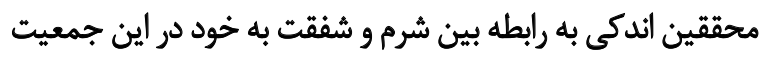

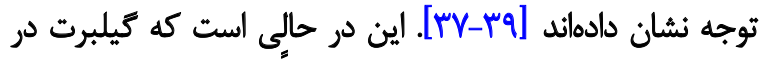

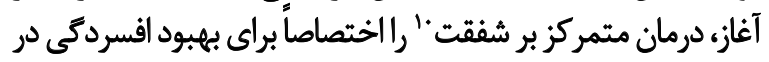

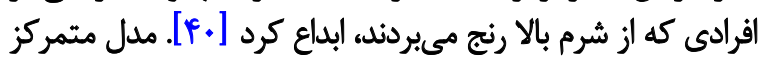

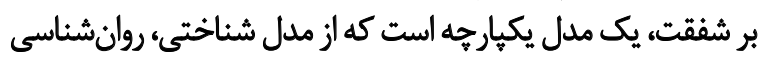

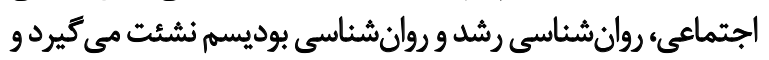

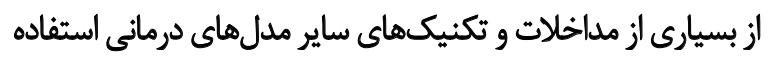

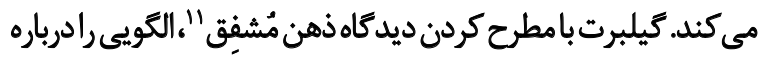

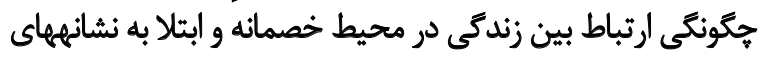

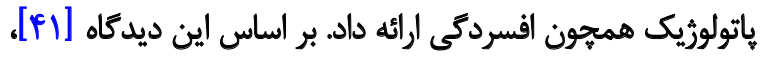

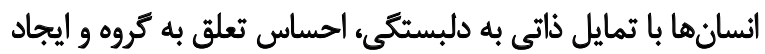

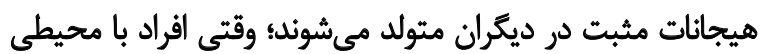

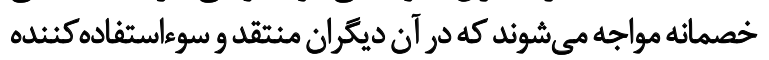

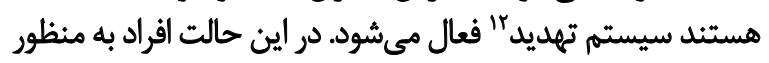

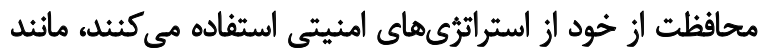

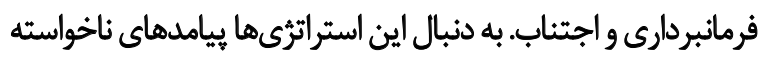

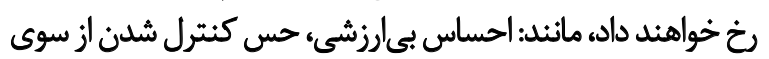

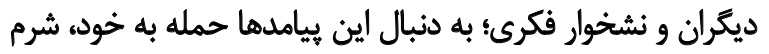

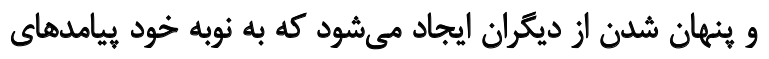

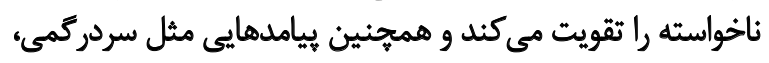

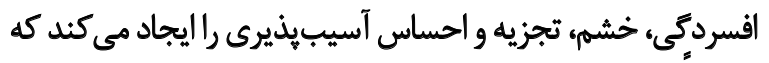

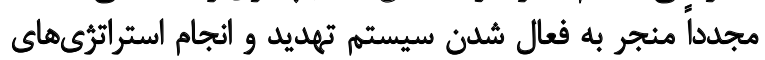

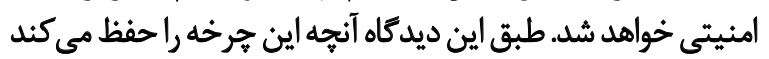

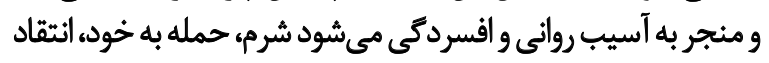

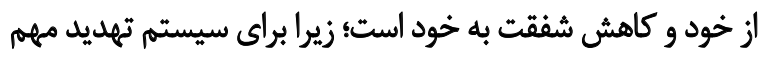

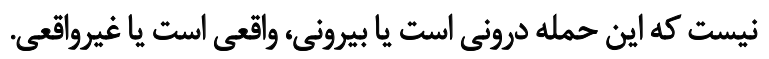

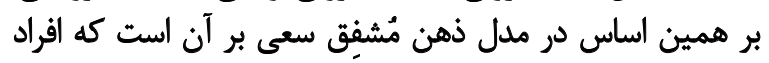

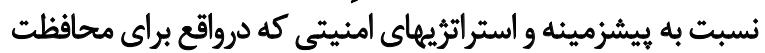

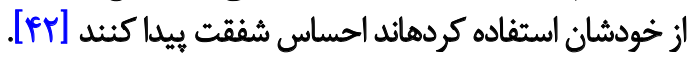

10. Compassion Focused Therapy (CFT)

11. Compassionate mind theory

12. Threat system 
مثياس شفقتث به خُود فرم كوثاه

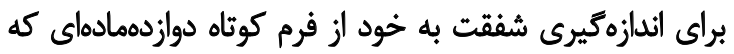

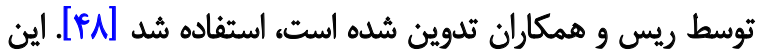

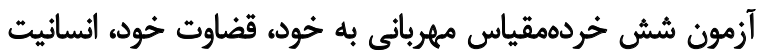

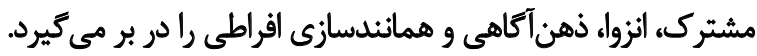

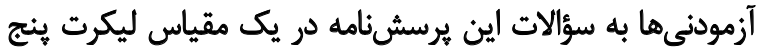

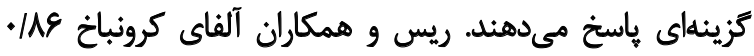

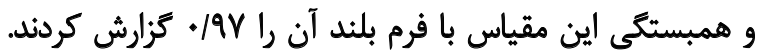

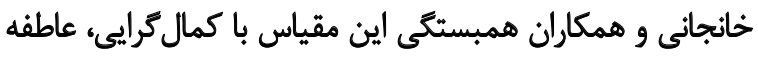

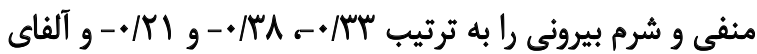

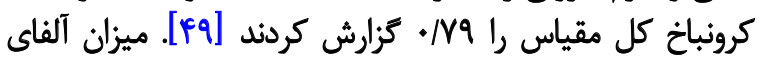

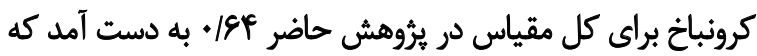
همجنان قابل قبول است.

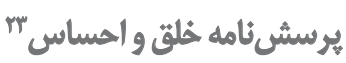

اين مقياس توسط انكلد و همكاران اختصاصاً براى جداسازي

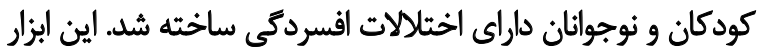

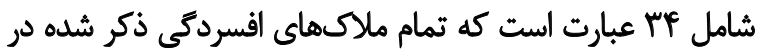

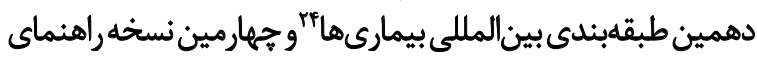

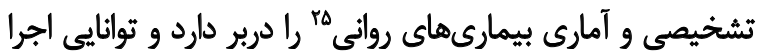

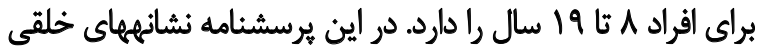

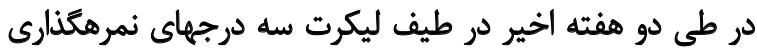

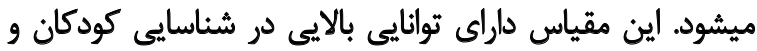

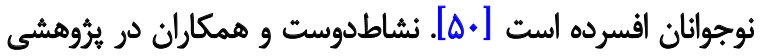

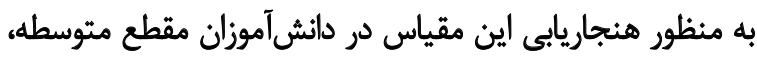

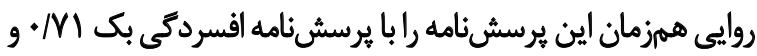

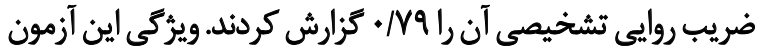

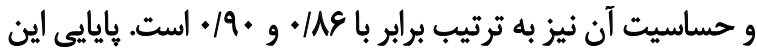

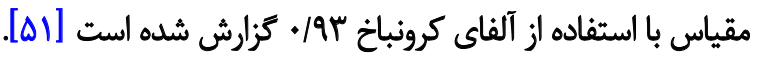

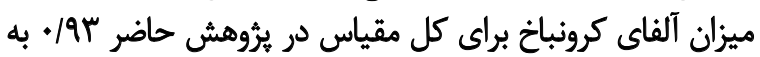

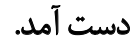

يافتهها

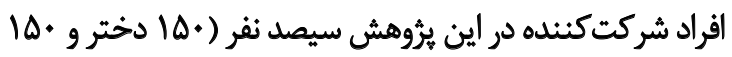

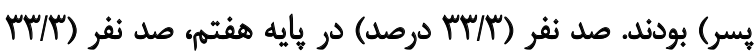

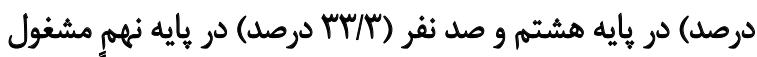

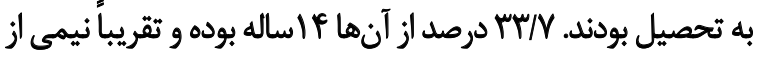

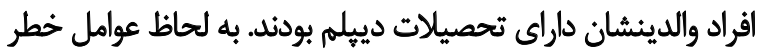

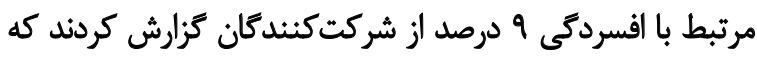

22. Self-Compassionate Scale-Short Form (SCS-SF)

23. Mood and Feeling Questionnaire (MFQ)

24. International Classification of Diseases, $10^{\text {th }}$ Edition [ICD-10] 25. Diagnostic and Statistical Manual of Mental Disorders, $4^{\text {th }}$ Edition [DSM-IV]
كودى محسوب مي شدئد، ابتدا رضايت والدين جلب شد. آنتاه هدف

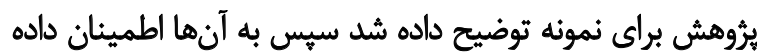

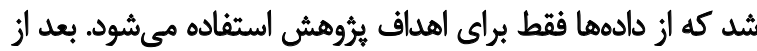

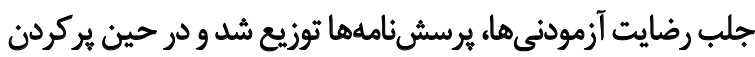

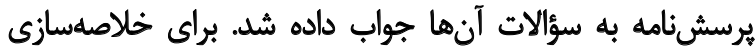

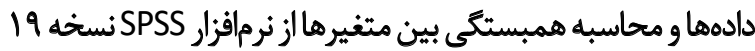

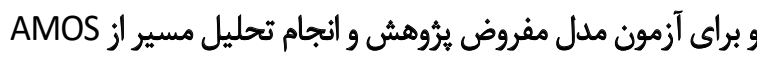

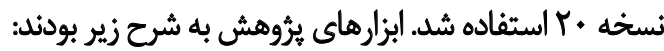

\section{مقياس قريانى يندبعدي"}

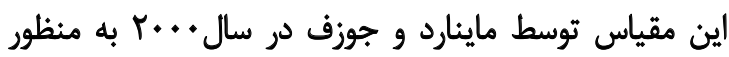

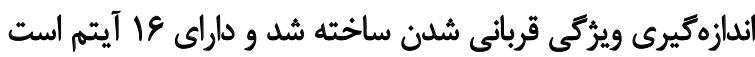

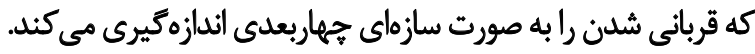

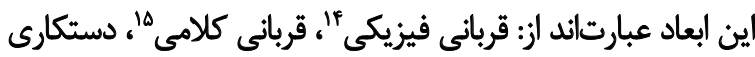

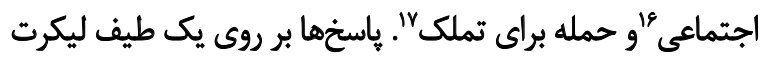

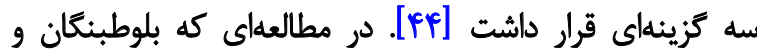

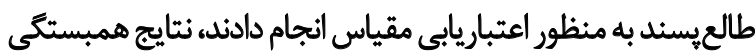

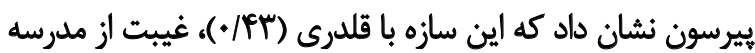

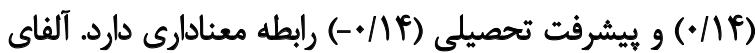

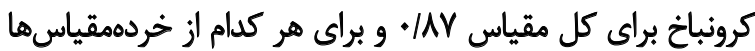

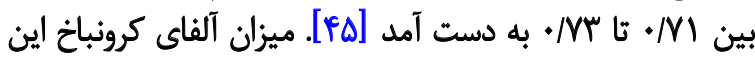

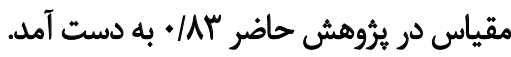

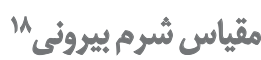

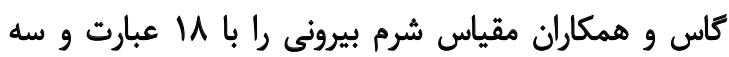

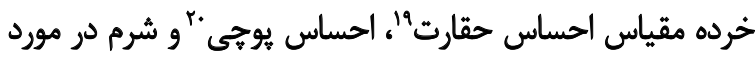

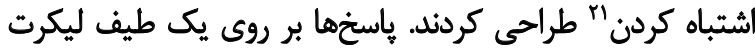

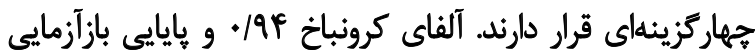

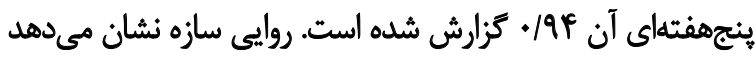

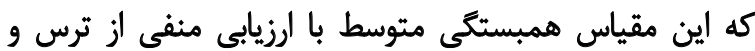

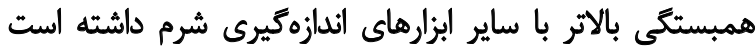

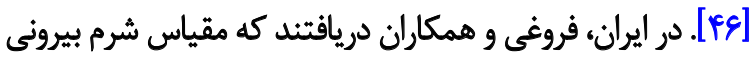

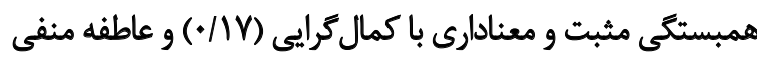

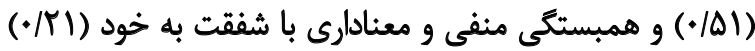

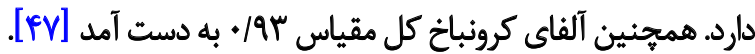

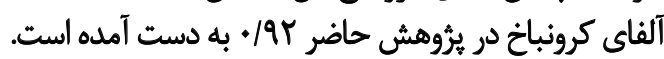

13. Multidimensional Peer-Victimization Scale (MPVS)

14. Physical Victimization

15. Verbal Victimization

16. Social manipulation

17. Attacks on property

18. The Other As Shamer Scale (OAS)

19. Inferiority

20. Emptiness

21. How others behave when they see me make mistakes 
جدول ا. شاخصهاى توصيفى متغيرهاى يثوهش

\begin{tabular}{|c|c|c|c|c|c|}
\hline كشيدكى & كجى & ميانتين ذانحراف استاندارد & بيشترين & كمترين & مثغير \\
\hline - /riva &.$/ 19$. & Nef \pm A/qf & m & - & قربائي شلن تونسط همرسالان \\
\hline $1 / 419$ & V/Far & $|f / \mu| \pm|r / \mu|$ & $n$ & • & شرم ييرونى \\
\hline$-+/ K M T$ & T & $\Psi N \Delta \cdot \pm V / \Delta \Delta$ & $\Delta \Delta$ & $M$ & شفقت به خود \\
\hline . /rar &.$/ 9 n$ & $1 \varepsilon / 4 a \pm 1 \% / V \varepsilon$ & $\Delta 9$ & - & نشانههاي افسردكى \\
\hline
\end{tabular}

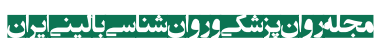

داده شدند. جدول شماره بّ ضرايب مسير و معنادارى آنها را نشان

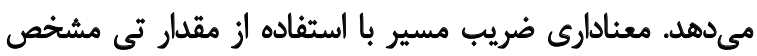

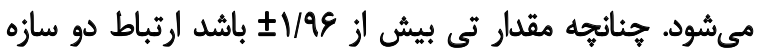

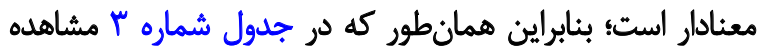

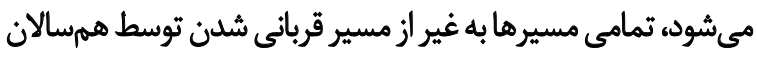

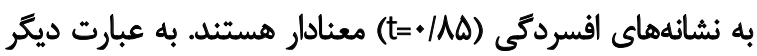

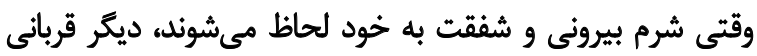

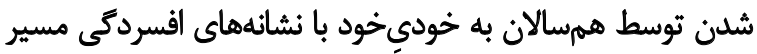

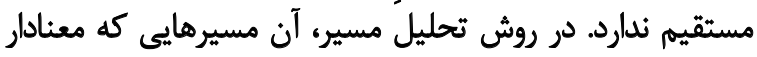

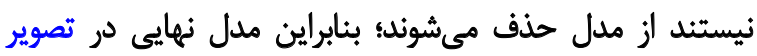

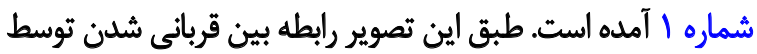

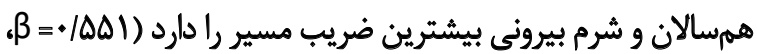

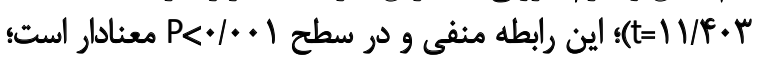

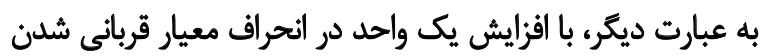

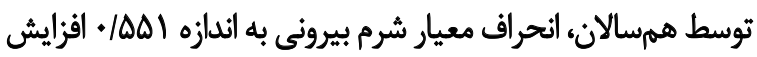

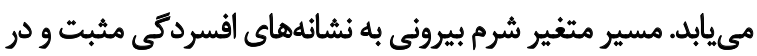

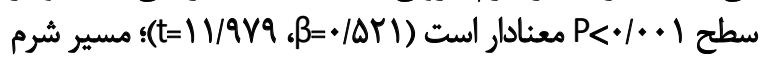

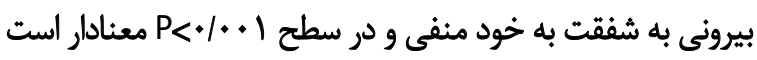

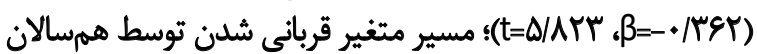

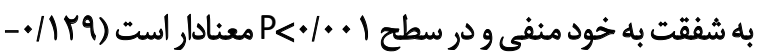

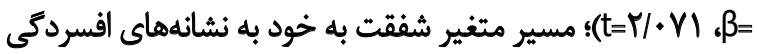

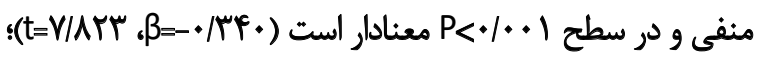

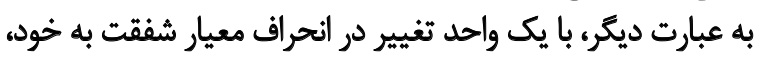

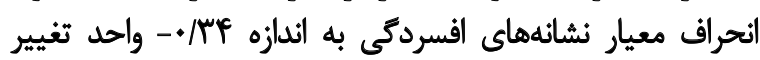

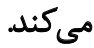

منظور از برازش مدل آن است كه تا جهه حد مدل فرأ فرضي بثروهش

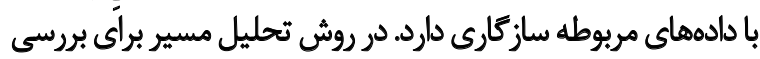

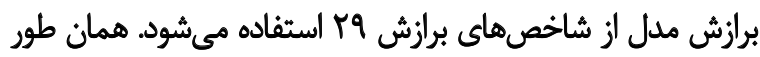

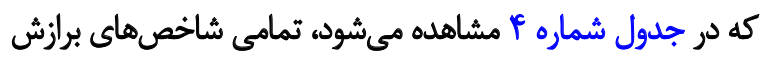

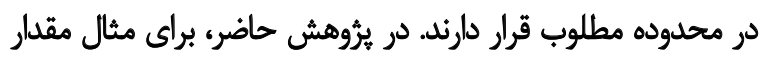

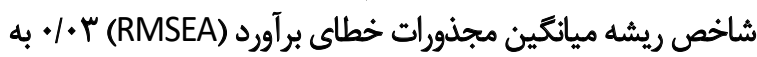

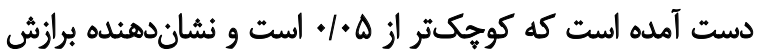

29. Fit indices
يكى از والدين سابقه بيمارى خلقى دارند، همجنين 9 درصد از آنها

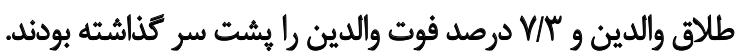

شاخصهاى توصيفى متغيرها در جدول شماره ا ديده مىشود.

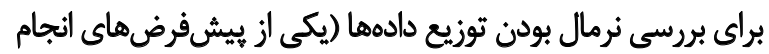

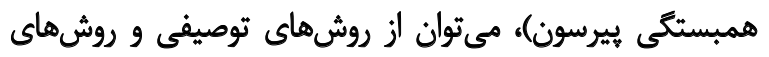

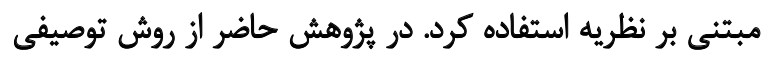

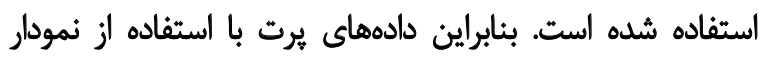

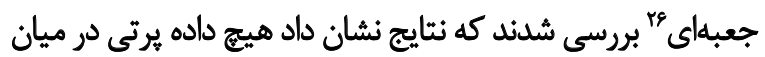

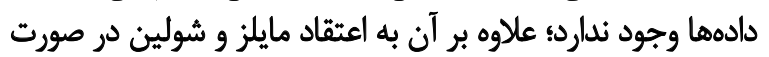

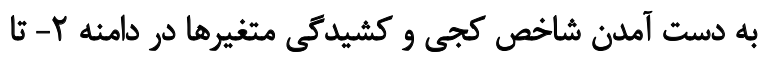

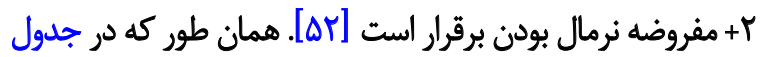

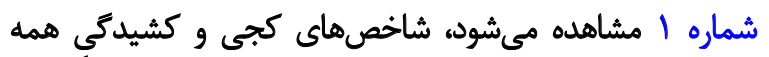

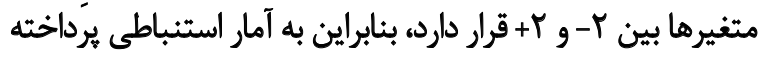
شد.

در جدول شماره T روابط بين متغيرهاي يُروهش نشان داده شده

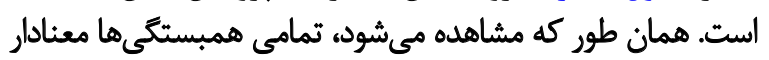

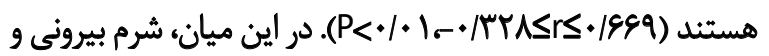

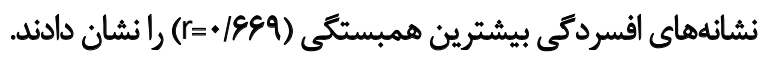

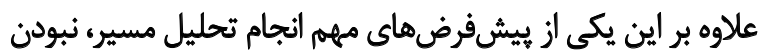

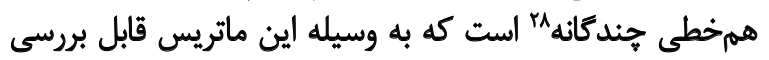

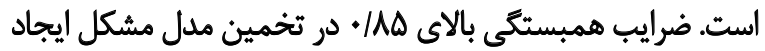

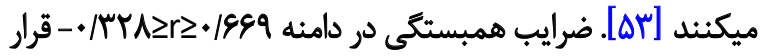

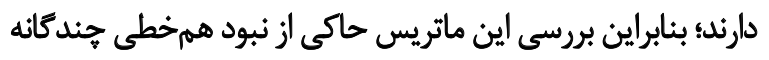
بين متغيرهاست. رسم مدل و بورسي مسير هاي مستثيثم بين متغيرها. بدين منظور متغيرها و مسيرهاى بين آنها به نرم|فزار

26. Boxplot

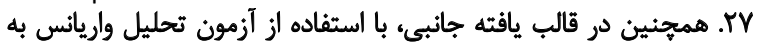

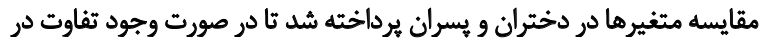

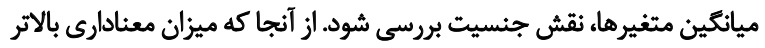

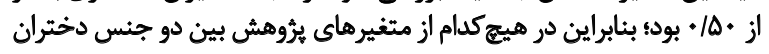

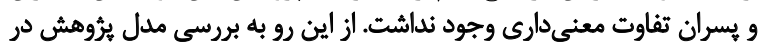

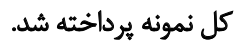

28. Multicollinearity 
جدول r. ماتريس همبستكى متغيرهاى ثئروهش

\begin{tabular}{|c|c|c|c|c|}
\hline 1 & $r$ & $r$ & $\varepsilon$ & متغير هاى يثروهش \\
\hline \multirow[t]{4}{*}{1} &.$|\Delta \Delta|^{* *}$ &.$- / M T \Lambda^{* *}$ &.$/ A T A^{* *}$ & 1. قربانى شلن توسط همرسالان \\
\hline & 1 & 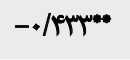 & +1899** & r. شرم بيرونى \\
\hline & & 1 & $-+1 \Delta \& \varphi^{*}$ & س. شفقت به خود \\
\hline & & & 1 & أ. نشانههاي اقسردكى \\
\hline
\end{tabular}

با ميانجي كُى شرم بيروني و شفقت به خود با ضريب استاندارد

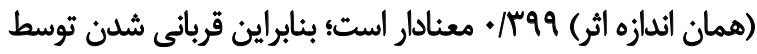

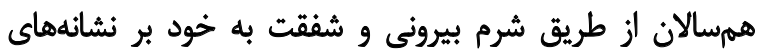

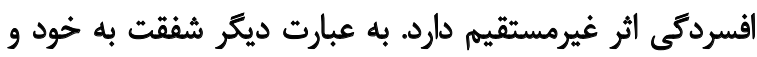

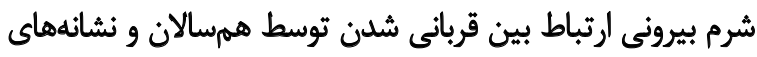

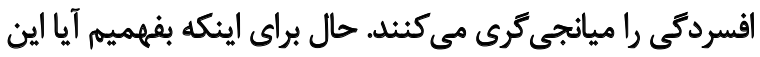

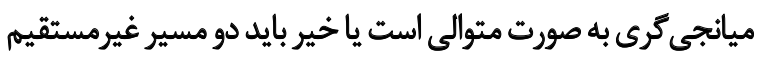
ديكر را بررسى كنيهم.

براى مسير قربانى شدن توسط همرسالان به شفقت به خود از

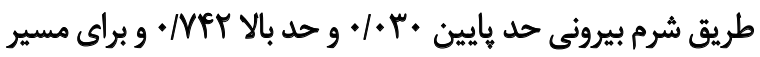

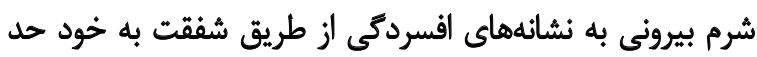

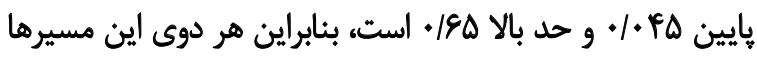

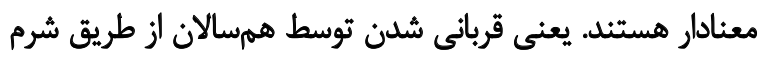

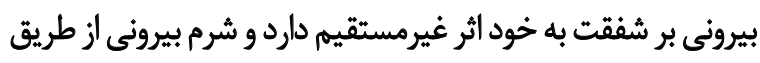

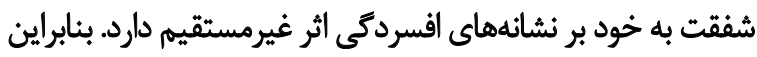

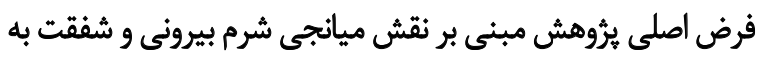

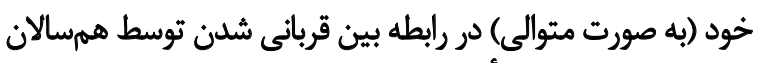

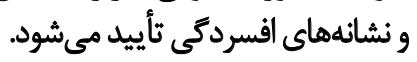

ثִ

هدف از اين مطالعه تعيين نقش ميانجى شرم بيرونى و شفقت به

مطلوب مدل است. از مجموع اين شاخص ها مي توان استنباط كرد كه

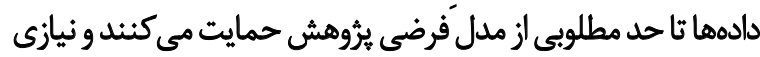
به اصلاح مدل نيست.

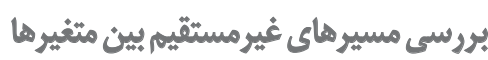

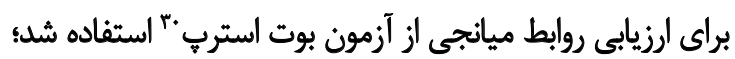

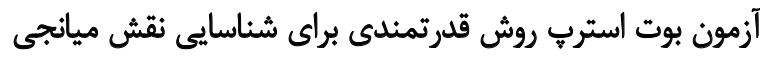

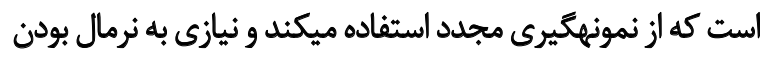

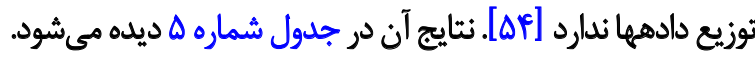

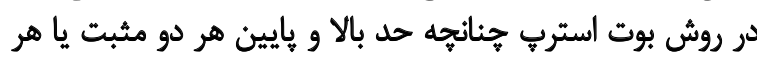

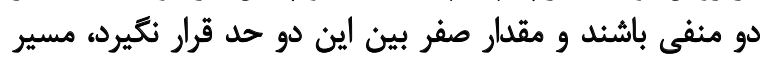

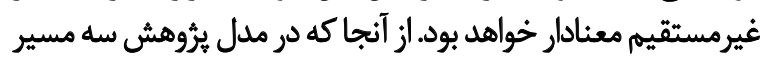

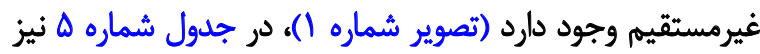

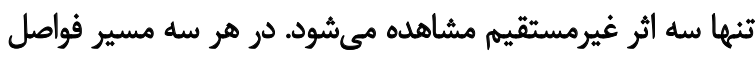

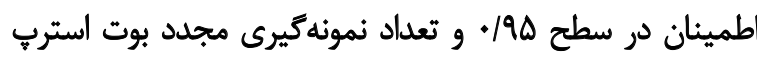

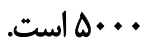

براى مسير قربانى شدن توسط همرالان به نشانههاى افسردكى

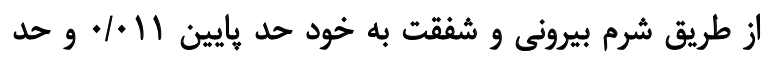

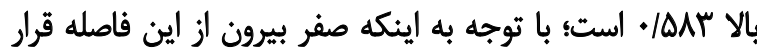

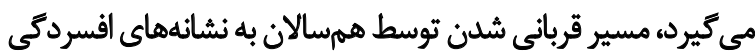

30. Bootstrap

جدول ب. بررسى ضرايب مسير بين متغيرهاي ثئوهش

\begin{tabular}{|c|c|c|c|}
\hline t (C.R.) & خُطاى اسثاندارد & ضريب استاندارد & متغير \\
\hline.$/ 10 r$ & $\cdot 1 \cdot 1$ & .1 .8 & قربائى شلن \\
\hline $11 / 4 \cdot r=0$ & $\cdot 11 \cdot 1$ &.$|\Delta \Delta|$ & قريانى شلن ـ شرم بيرونى \\
\hline 11/979*** & $.1+4 \Delta$ &.$|\Delta T|$ & شرم بيرونى ـ افسردكى \\
\hline -Q/ArT"en & $.1 \cdot 4 \Delta$ &.$- /$ MET & شرم بيرونى ـ شفقت به خود \\
\hline$-r / \cdot V)^{*}$ & $\cdot 1 \cdot \sqrt{9}$ &.$- / 17 q$ & قرباني شلن ك شققت به خُود \\
\hline -V/ArT" & $1+v 9$ &,$- / M F$ & شفقت به خود ـ افسردكى \\
\hline
\end{tabular}




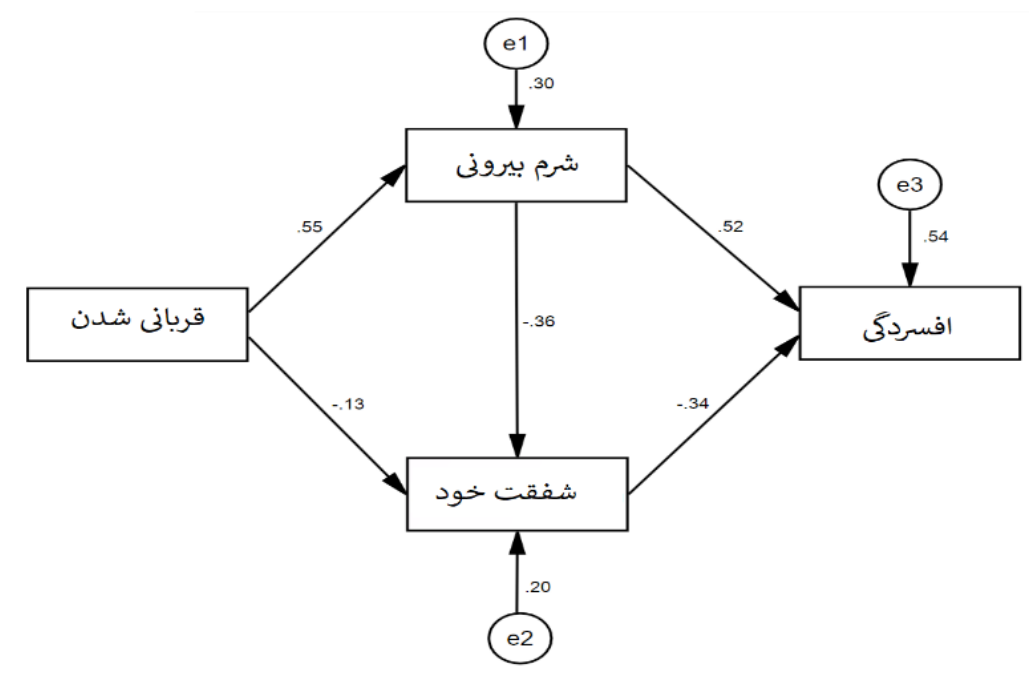

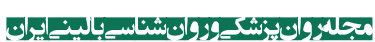

تصوير ا. مدل فرضى يروهش به همراه ضريب استاندارد مسيرها بررسى برازش مدل

شرم مىثواند از طريق روابط همسالان و به خصوص قربانى شدن

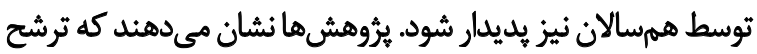

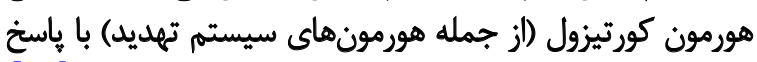

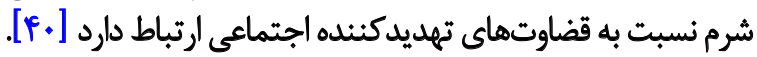

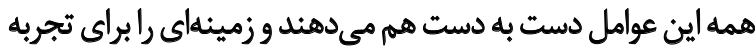

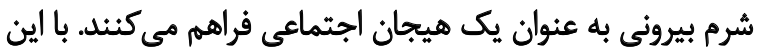

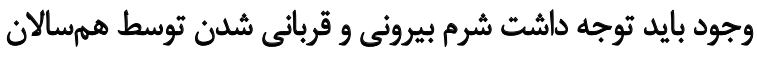

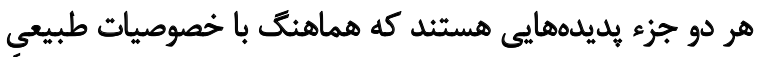
اين دوره افزايش مى يابند.

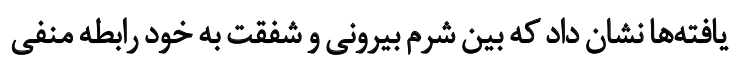

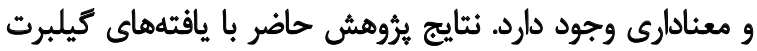

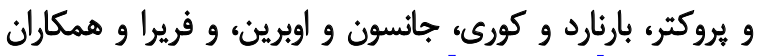

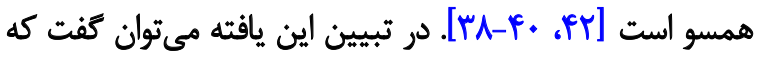

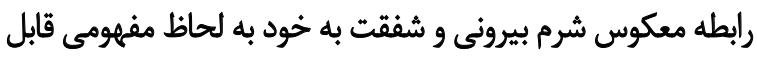

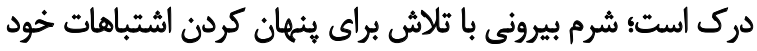

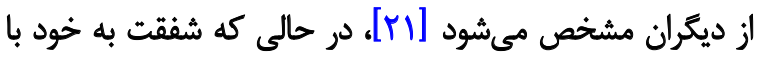

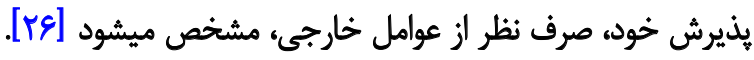

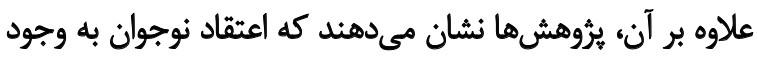

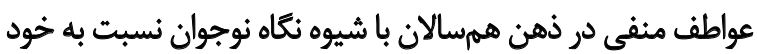

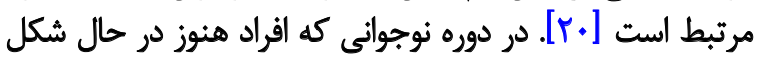

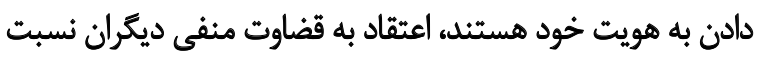

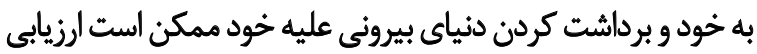

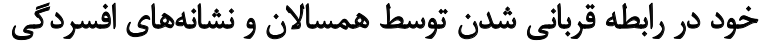

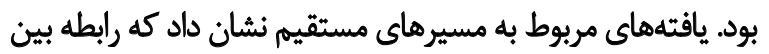

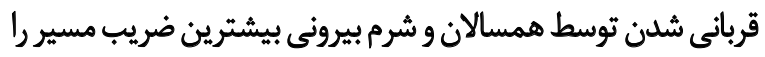

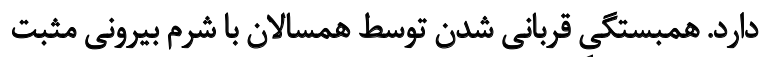

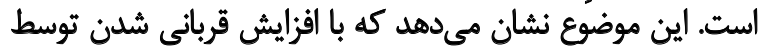

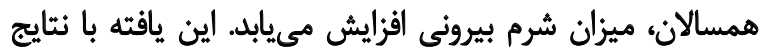

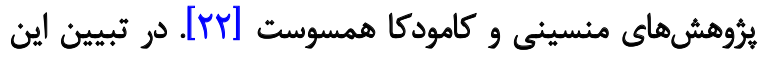

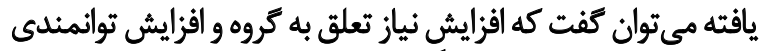

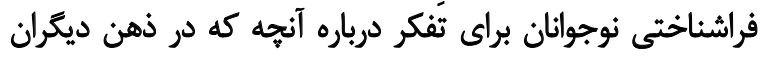

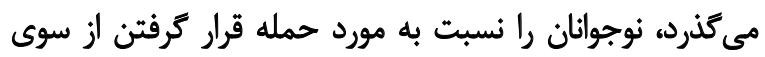

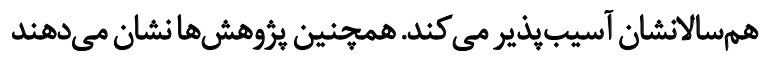

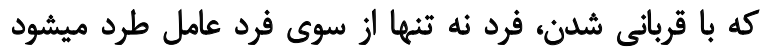

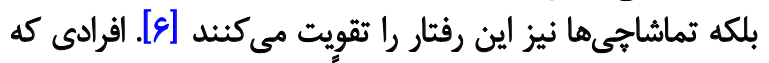

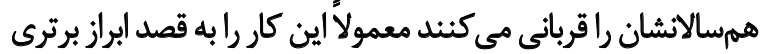

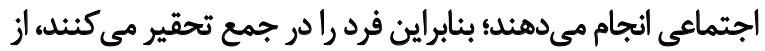

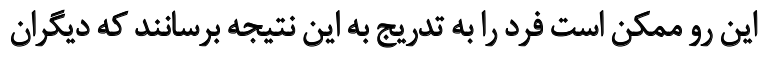

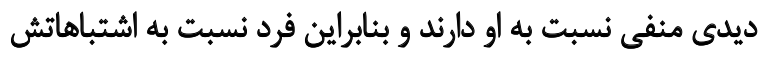

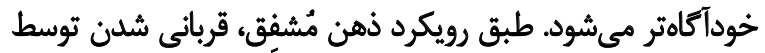

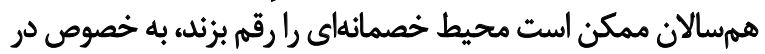

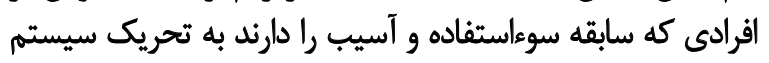

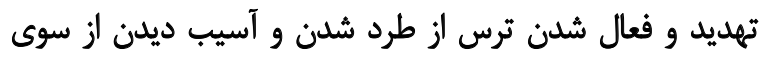

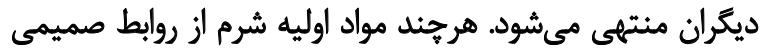

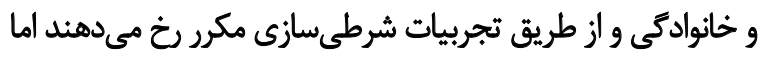

جدول F. شاخصهاى برازش مدل فرضى ئروهش

\begin{tabular}{|c|c|c|c|c|c|c|c|c|c|}
\hline PGFI & AGFI & GFI & RFI & CFI & TLI & NFI & RMSEA & $x^{Y} / d f$ & شاخصها \\
\hline .1999 &.$/ 9 \vee 8$ & .194 & . $/ 979$ & .1999 &.$/ 997$ &.$/ 994$ & $.1+m$ & I/FIA & مقدار به دست أمده \\
\hline$>+190$ & $>\cdot / 9 \Delta$ & $>\cdot 190$ & $>. / 9 \Delta$ & $>\cdot 190$ & $>* / 9$ & $>\cdot 190$ & $<+1+\infty$ & $<r$ & بازه مطلوب \\
\hline
\end{tabular}

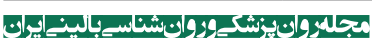


جدول هـ نتايج آزمون بوت استرب براى مسيرهاى غيرمستقيم

\begin{tabular}{|c|c|c|c|c|c|c|}
\hline قاصله اطمينان & اندازه اثر & حد بالا & حد هايين & ملاك & ميانجى & مثغير ييشبين \\
\hline .190 & . & / $/ Q A T$ & $.1+11$ & افسردكى & شرم بيرونى وشفقت به خود & قربانى شلن \\
\hline.$/ 9 \Delta$ &.$- / 199$ & . Mer & 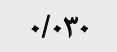 & شفقت به خود & شرم بيرونى & قربانى شلن \\
\hline.$/ 9 \Delta$ & תואות &.$/ 8 \Delta$. & $.1 .4 \Delta$ & افسرد5ى & شفقت به خود & شرم بيرونى \\
\hline
\end{tabular}

به بيامد ناخواسته حمله به خود منتهيى شود كه شفقت نسبت به

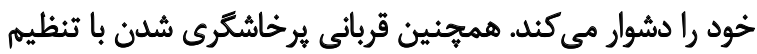

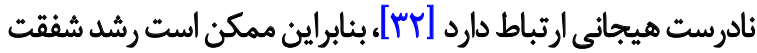

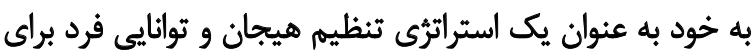

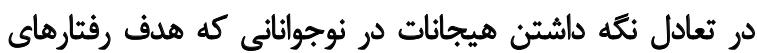

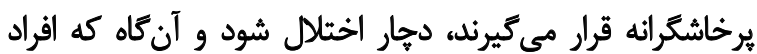

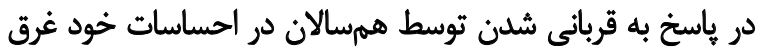

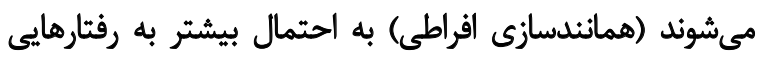

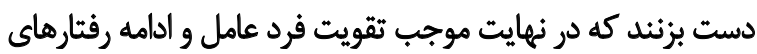

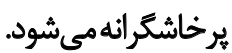

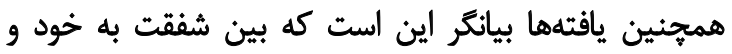

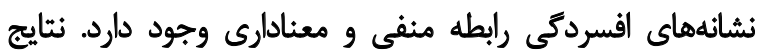

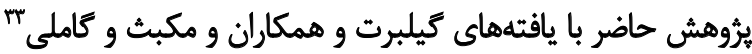

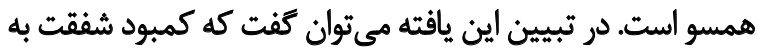

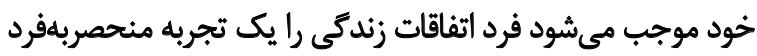

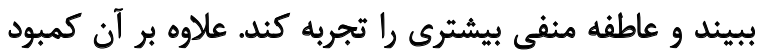

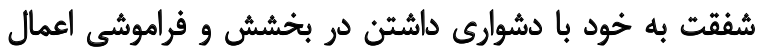

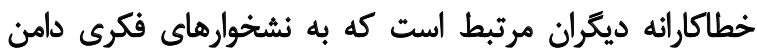

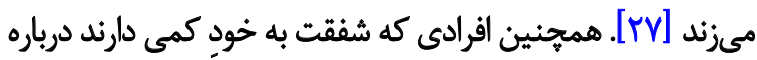

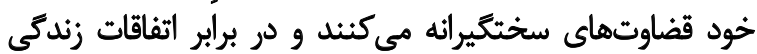

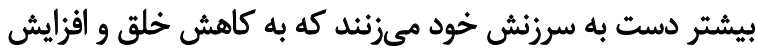

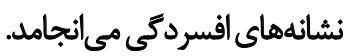

نتايج مرتبط با مسيرهاى غيرمستقيم نشان داد كه قربانى شلن

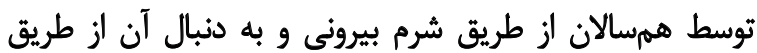

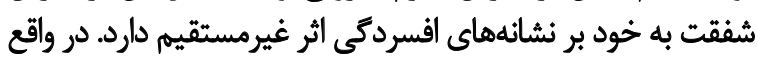

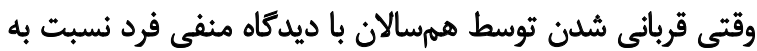

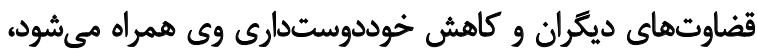

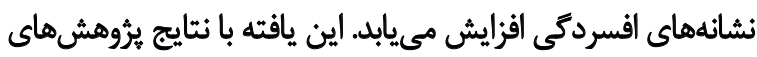

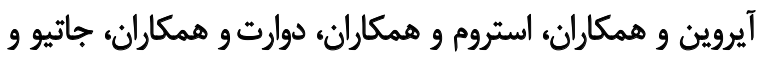

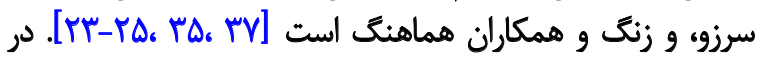

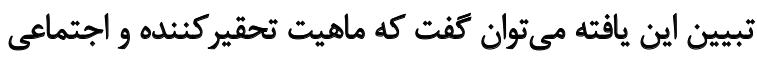

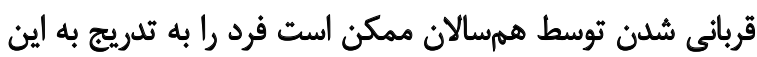

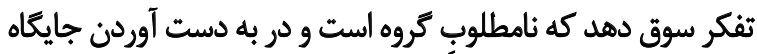

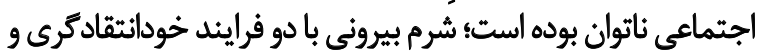

33. MacBeth and Gumley

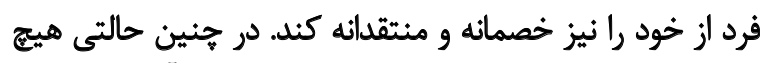

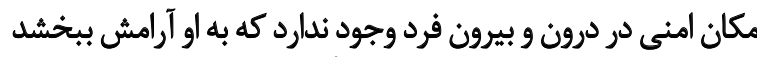

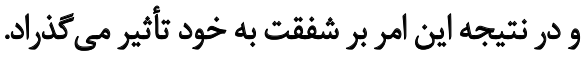

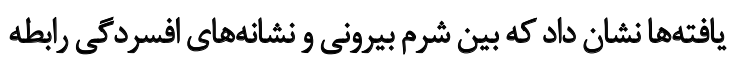

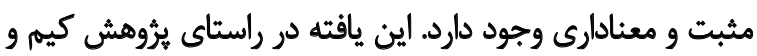

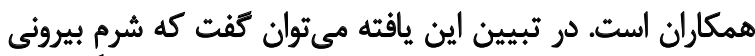

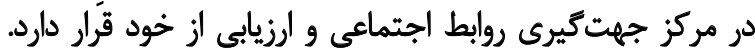

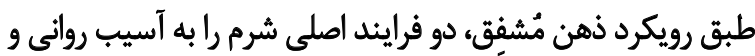

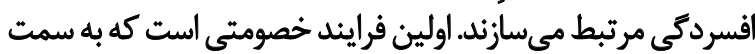

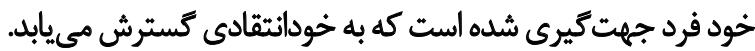

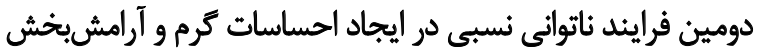

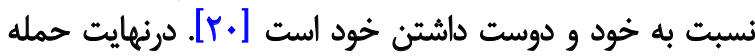

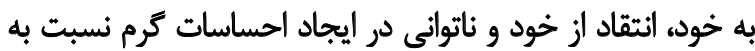

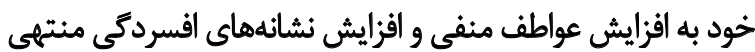

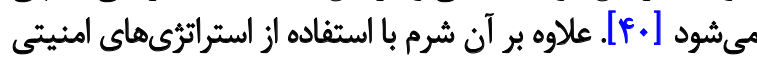

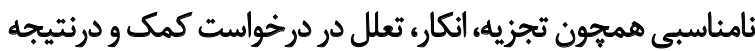

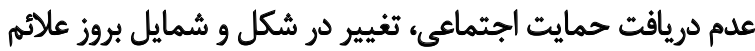

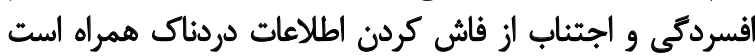

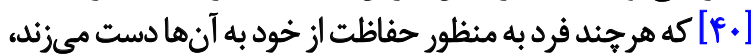

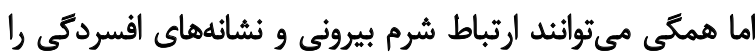

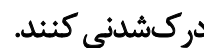

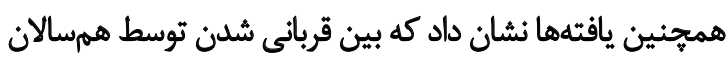

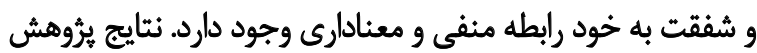

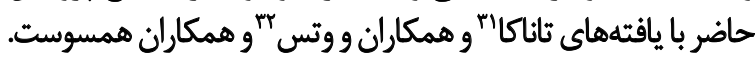

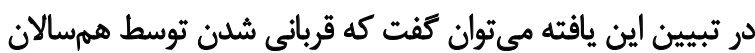

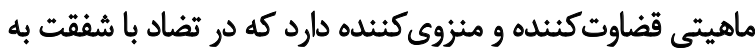

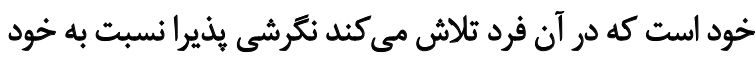

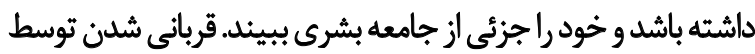

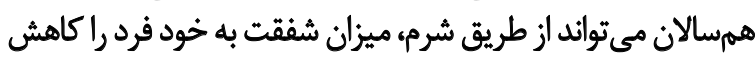

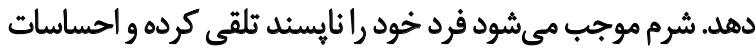

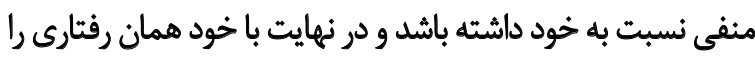

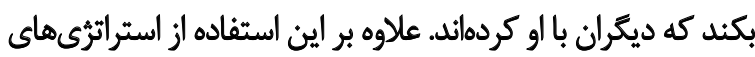

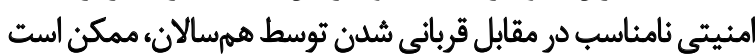


برؤوهش حاضر فقط به اندازهميرى نوع سنتى قربانى شدن

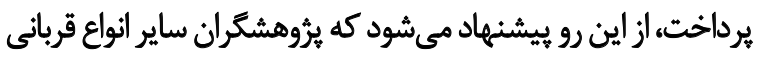

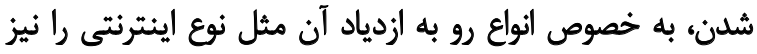

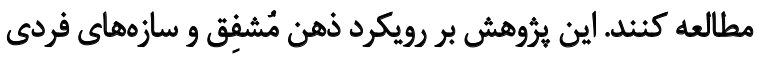

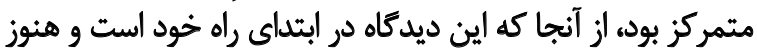

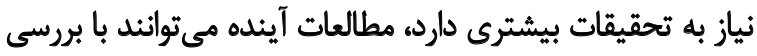

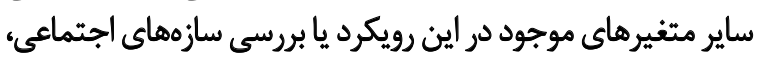

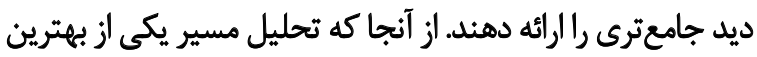

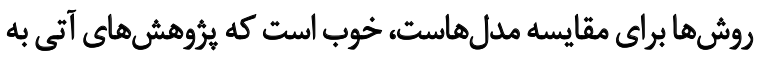

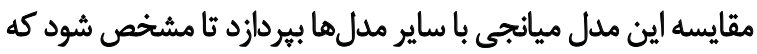
كدام مدل نظرى شواهد قوى ترى را فراين ماهم مي آورد.

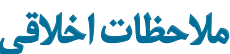 \\ ي بيروى أز اصول اخلاق يثونش}

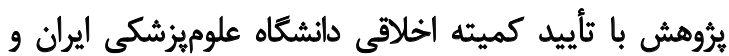

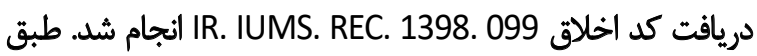

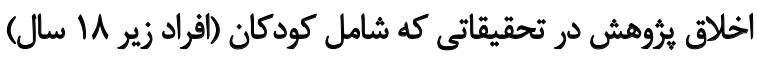

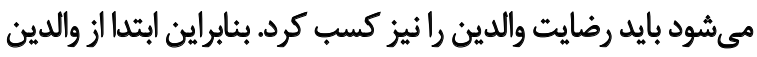

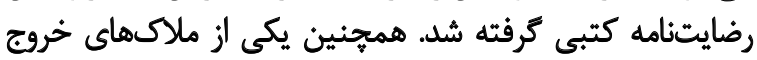

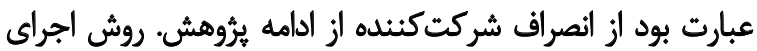

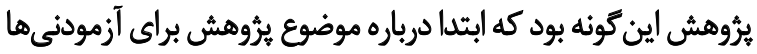

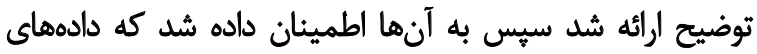

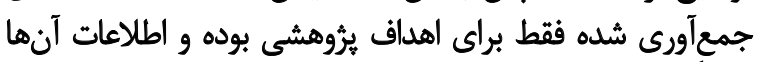

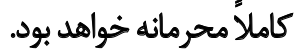

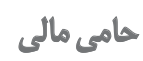

اين تحقيق هيج كونه كمك مالى از سازمانهاي تأمين مالى در بخشهاى عمومى ، تجارى يا غيرانتفاعى دريافت نكرد.

$$
\text { هشاركت نويسيندكان }
$$

مفهومسازي:همهنويسندكان؛ روش شناسى: سهيلايعقوبى واحمد

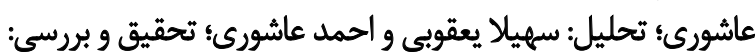

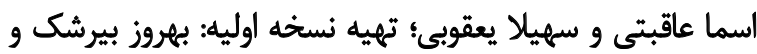

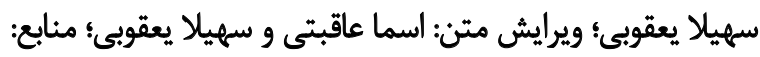

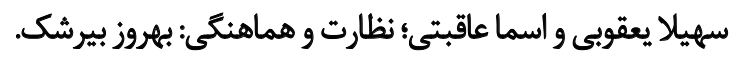

$$
\text { تعارض مناقع }
$$

بنابر اظهار نويسندكان اين مقاله تعارض منافع ندارد.

$$
\text { تشكر و قدر داني }
$$

از اداره آموزش و يرورش استان اصفهان و همجنين اداره آموزش و يرورش شهر شاهين شهر و تمامى شركت كنئدانان تشكر مى كنيه.

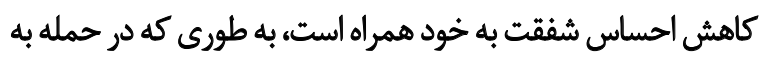

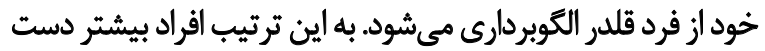

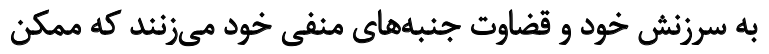

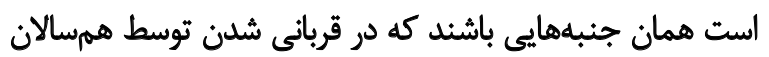

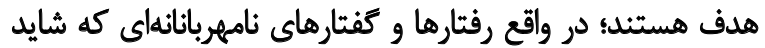

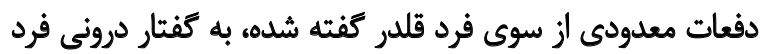

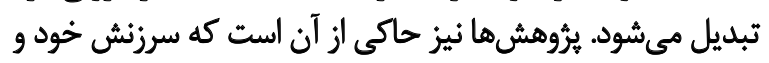

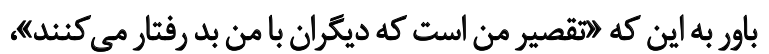

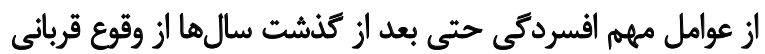

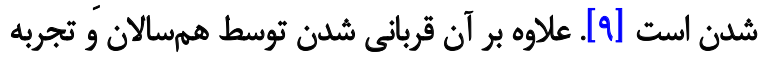

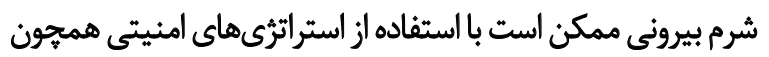

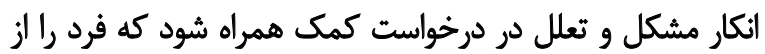

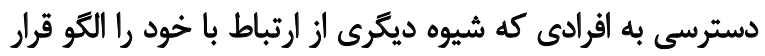

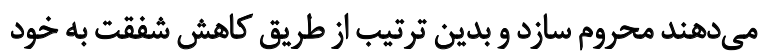
به تشديد نشانههاى افسردخى كمك كنيند.

\section{نتيجنيّيرى}

يافتهها تأييدكننده نقش و اهميت شرم بيرونى و شفقت به خود

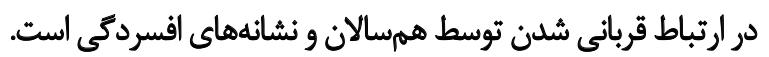

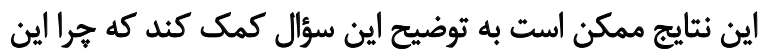

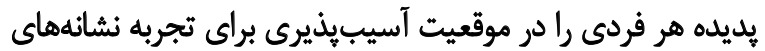

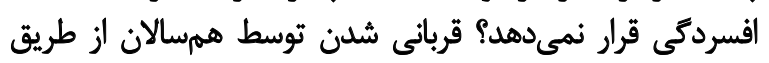

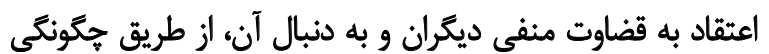

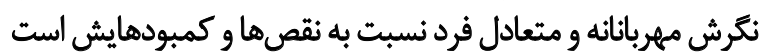

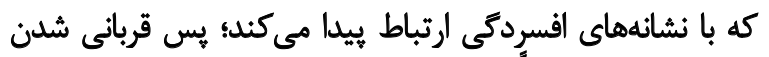

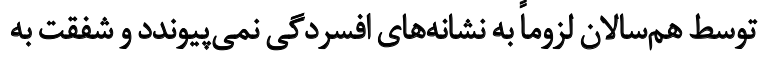

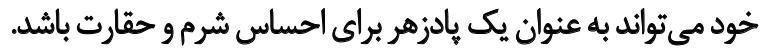

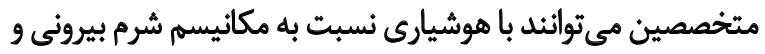

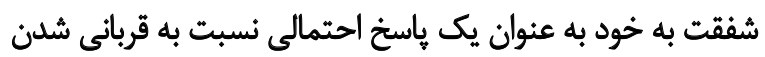

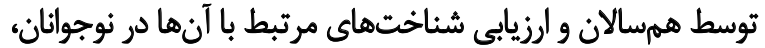

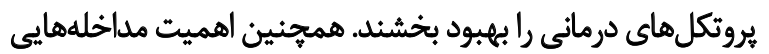

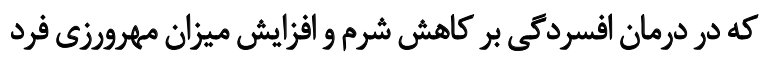

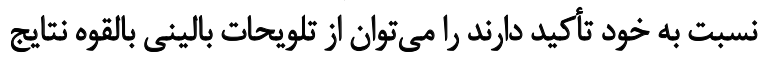
اين يُروهش در نظر ترفت.

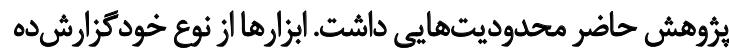

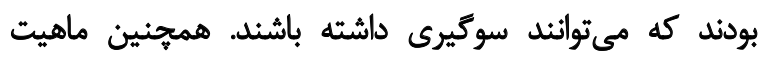

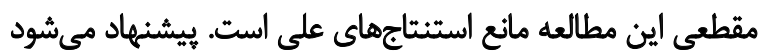

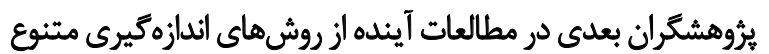

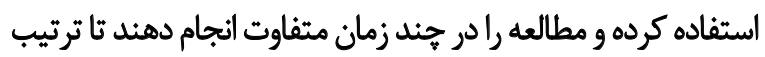

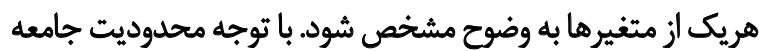

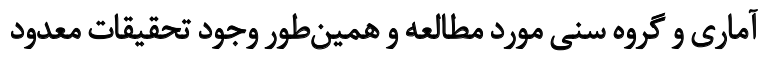

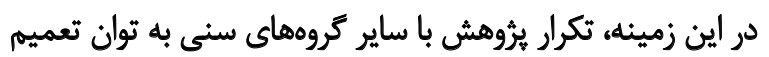
يافتهها كمك خواهد كرد. 


\section{References}

[1] Galambos NL, Leadbeater BJ, Barker ET. Gender differences in and risk factors for depression in adolescence: A 4-year longitudinal study. International Journal of Behavioral Development. 2004; 28(1):16-25. [DOI:10.1080/01650250344000235]

[2] Swearer SM, Hymel S. Understanding the psychology of bullying: Moving toward a social-ecological diathesis-stress model. American Psychologist. 2015; 70(4):344. [DOI:10.1037/a0038929] [PMID]

[3] Finkelhor D, Turner HA, Hamby S. Let's prevent peer victimization, not just bullying. Child Abuse \& Neglect. 2012; 36(4):271-4. [DOI:10.1016/j.chiabu.2011.12.001] [PMID]

[4] Hawker DS, Boulton MJ. Twenty years' research on peer victimization and psychosocial maladjustment: A meta-analytic review of cross-sectional studies. Journal of Child Psychology and Psychiatry. 2000; 41(4):441-55. [DOI:10.1111/1469-7610.00629] [PMID]

[5] Troop-Gordon W. Peer victimization in adolescence: The nature, progression, and consequences of being bullied within a developmental context. Journal of Adolescence. 2017; 55:116-28. [DOI:10.1016/j.adolescence.2016.12.012] [PMID]

[6] Mazaheri Tehrani MA, Shiri S, Valipour M. [Studying nature and prevalence of bullying in zanjan's rural secondary schools (Persian)]. Educational Psychology. 2015; 11(36):17-38. https:// jep.atu.ac.ir/article_1588_en.html

[7] Nylund K, Bellmore A, Nishina A, Graham S. Subtypes, severity, and structural stability of peer victimization: What does latent class analysis say?. Child Development. 2007; 78(6):1706-22. [DOI:10.1111/j.1467-8624.2007.01097.x] [PMID]

[8] Copeland WE, Wolke D, Angold A, Costello EJ. Adult psychiatric outcomes of bullying and being bullied by peers in childhood and adolescence. JAMA Psychiatry. 2013; 70(4):419-26. [DOI:10.1001/jamapsychiatry.2013.504] [PMID] [PMCID]

[9] Bowes L, Joinson C, Wolke D, Lewis G. Peer victimisation during adolescence and its impact on depression in early adulthood: prospective cohort study in the United Kingdom. The BMJ. 2015; 350. [DOI:10.1136/bmj.h2469] [PMID] [PMCID]

[10] Cairns KE, Yap MB, Reavley NJ, Jorm AF. Identifying prevention strategies for adolescents to reduce their risk of depression: A Delphi consensus study. Journal of affective disorders. 2015; 183:229-38.[DOI:10.1016/j.jad.2015.05.019] [PMID]

[11] Brendgen M, Poulin F, Denault AS. Peer victimization in school and mental and physical health problems in young adulthood: Examining the role of revictimization at the workplace. Developmental Psychology. 2019; 55(10):2219-30. [DOI:10.1037/ dev0000771] [PMID]

[12] Lotfi S, Dolatshahi B, Mohammadkhani P, Campbell M, Dogaheh ER. [Prevalence of bullying and its relationship with trauma symptoms in young Iranian students (Persian)]. Journal of Practice in Clinical Psychology. 2014; 2(4):271-6. https:/ / eprints.qut. edu.au/83700/

[13] Ebrahimi A, Hakim Shoshtari M, Asgharzade A, Karsazi H. [Depression-anxiety symptoms and its relatinship with childhood teasing experiences (Persian)]. Thought \& Behavior in Clinical Psychology. 2017; 12(45):27-37. https://jtbcp.riau.ac.ir/article _1151_82443d3740e2e179dd3df6bfd905df10.pdf
[14] Alba J, Calvete E, Wante L, Van Beveren ML, Braet C. Early maladaptive schemas as moderators of the association between bullying victimization and depressive symptoms in adolescents. Cognitive Therapy and Research. 2018; 42(1):24-35. [DOI:10.1007/ s10608-017-9874-5]

[15] Baker OE, Bugay A. Peer victimization and depressive symptoms: The mediation role of loneliness. Procedia Social and Behavioral Sciences. 2011; 30:1303-7. [DOI:10.1016/j.sbspro.2011.10.253]

[16] Du C, DeGuisto K, Albright J, Alrehaili SJIJoPS. Peer support as a mediator between bullying victimization and depression International Journal of Psychological Studies. 2018; 10(1):59. [DOI:10.5539/ijps.v10n1p59]

[17] Chu XW, Fan CY, Liu QQ, Zhou ZK. Cyberbullying victimization and symptoms of depression and anxiety among Chinese adolescents: Examining hopelessness as a mediator and self-compassion as a moderator. Computers in Human Behavior. 2018; 86:377-86. [DOI:10.1016/j.chb.2018.04.039]

[18] Zhou ZK, Liu QQ, Niu GF, Sun XJ, Fan CY. Bullying victimization and depression in Chinese children: A moderated mediation model of resilience and mindfulness. Personality and Individual Differences. 2017; 104:137-42. [DOI:10.1016/j.paid.2016.07.040]

[19] Gilbert P. The evolution of shame as marker for relationship security. In: Tracy J, Robins RW, Tangney JP, editors. The SelfConscious Emotions Theory and Research. New York: Guilford; 2007.

[20] Gilbert P, Irons C. Shame, self-criticism, and self-compassion in adolescence. In: Sheeber LB, Allen NB, editors. Adolescent Emotional Development and the Emergence of Depressive Disorders. Cambridge: Cambridge University Press; 2009. [DOI:10.1017/ CBO9780511551963.011]

[21] Kim S, Thibodeau R, Jorgensen RS. Shame, guilt, and depressive symptoms: A meta analytic review. Psychological Bulletin. 2011; 137(1):68. [DOI:10.1037/a0021466] [PMID]

[22] Menesini E, Camodeca M. Shame and guilt as behaviour regulators: Relationships with bullying, victimization and prosocial behaviour. British Journal of Developmental Psychology. 2008; 26(2):183-96. [DOI:10.1348/026151007X205281]

[23] Irwin A, Li J, Craig W, Hollenstein T. The role of shame in the relation between peer victimization and mental health outcomes. Journal of Interpersonal Violence. 2019; 34(1):156-81 [DOI:10.1177/0886260516672937] [PMID]

[24] Strøm IF, Aakvaag HF, Birkeland MS, Felix E, Thoresen S. The mediating role of shame in the relationship between childhood bullying victimization and adult psychosocial adjustment. European Journal of Psychotraumatology. 2018; 9(1):1418570. [DOI:10. 1080/20008198.2017.1418570] [PMID] [PMCID]

[25] Duarte C, Pinto-Gouveia J, Rodrigues T. Being bullied and feeling ashamed: Implications for eating psychopathology and depression in adolescent girls. Journal of Adolescence. 2015 44:259-68. [DOI:10.1016/j.adolescence.2015.08.005] [PMID]

[26] Neff K. Self-compassion: An alternative conceptualization of a healthy attitude toward oneself. Self and Identity. 2003; 2(2):85101. [DOI:10.1080/15298860309032]

[27] Yaghoubi S, Akrami N. [Role of Self-Compassion in Prediction of Forgiveness and Empathy in Young Adults (Persian)]. Positive Psychology Research. 2016; 2(3):35-48. http://ensani.ir/file/ download/article/1560860673-10047-95-15.pdf 
[28] Bluth K, Mullarkey M, Lathren C. Self-compassion: A potential path to adolescent resilience and positive exploration. Journal of Child and Family Studies. 2018; 27(9):3037-47.[DOI:10.1007/ s10826-018-1125-1]

[29] MacBeth A, Gumley A. Exploring compassion: A meta-analysis of the association between self-compassion and psychopathology. Clinical Psychology Review. 2012; 32(6):545-52. [DOI:10.1016/j. cpr.2012.06.003] [PMID]

[30] Neff KD, Vonk R. Self-compassion versus global self-esteem: Two different ways of relating to oneself. Journal of Personality. 2009; 77(1):23-50. [DOI:10.1080/15298860309027]

[31] Bagheri J, Birashk B, Dehghani M, Asgharnejad AA. The relationship between object relations and the severity of depression symptoms: The mediating role of self-compassion. Iranian Journal of Psychiatry and Clinical Psychology. 2019; 25(3):328-43. [DOI:10.32598/ijpcp.25.3.328]

[32] Gratz KL, Tull MT, Baruch DE, Bornovalova MA, Lejuez CW. Factors associated with co-occurring borderline personality disorder among inner-city substance users: The roles of childhood maltreatment, negative affect intensity/reactivity, and emotion dysregulation. Comprehensive Psychiatry. 2008; 49(6):603-15. [DOI:10.1016/j.comppsych.2008.04.005] [PMID]

[33] Vettese LC, Dyer CE, Li WL, Wekerle C. Does self-compassion mitigate the association between childhood maltreatment and later emotion regulation difficulties? A preliminary investigation. International Journal of Mental Health and Addiction. 2011; 9(5):480-91. [DOI:10.1007/s11469-011-9340-7]

[34] Tanaka M, Wekerle C, Schmuck ML, Paglia-Boak A, MAP Research Team. The linkages among childhood maltreatment, adolescent mental health, and self-compassion in child welfare adolescents. Child Abuse \& Neglect. 2011; 35(10):887-98. [DOI:10.1016/j.chiabu.2011.07.003] [PMID]

[35] Játiva R, Cerezo MA. The mediating role of self-compassion in the relationship between victimization and psychological maladjustment in a sample of adolescents. Child abuse \& Neglect. 2014; 38(7):1180-90. [DOI:10.1016/j.chiabu.2014.04.005] [PMID]

[36] Ttofi MM, Farrington DP. Effectiveness of school-based programs to reduce bullying: A systematic and meta-analytic review. Journal of Experimental Criminology. 2011; 7(1):27-56. [DOI:10.1007/s11292-010-9109-1]

[37] Zhang H, Carr ER, Garcia-Williams AG, Siegelman AE, Berke D, Niles-Carnes LV, Patterson B, Watson-Singleton NN, Kaslow NJ. Shame and depressive symptoms: Self-compassion and contingent self-worth as mediators? Journal of Clinical Psychology in Medical Settings. 2018; 25(4):408-19. [DOI:10.1007/s10880-0189548-9] [PMID]

[38] Barnard LK, Curry JF. The relationship of clergy burnout to self-compassion and other personality dimensions. Pastoral Psychology. 2012; 61(2):149-63. [DOI:10.1007/s11089-011-0377-0]

[39] Ferreira C, Pinto-Gouveia J, Duarte C. Self-compassion in the face of shame and body image dissatisfaction: Implications for eating disorders. Eating Behaviors. 2013; 14(2):207-10. [DOI:10.1016/j.eatbeh.2013.01.005] [PMID]

[40] Gilbert P, Procter S. Compassionate mind training for people with high shame and self-criticism: Overview and pilot study of a group therapy approach. Clinical Psychology \& Psychotherapy: An International Journal of Theory \& Practice. 2006; 13(6):353-79. [DOI:10.1002/cpp.507]
[41] Gilbert P. An introduction to compassion focused therapy in cognitive behavior therapy. International Journal of Cognitive Therapy. 2010;3(2):97-112. [DOI:10.1521/ijct.2010.3.2.97]

[42] Johnson EA, O'Brien KA. Self-compassion soothes the savage ego-threat system: Effects on negative affect, shame, rumination, and depressive symptoms. Journal of Social and Clinical Psychology. 2013; 32(9):939-63. [DOI:10.1521/jscp.2013.32.9.939]

[43] Streiner DL. Finding our way: an introduction to path analysis. The Canadian Journal of Psychiatry. 2005; 50(2):115-22. [DOI:10.1 177/070674370505000207] [PMID]

[44] Mynard H, Joseph S. Development of the multidimensional peer-victimization scale. Aggressive Behavior: Official Journal of the International Society for Research on Aggression. 2000; 26(2):169-78. [DOI:10.1002/(SICI)1098-2337(2000)26:23.0.CO;2-A]

[45] Balootbangan AA, Talepasand SI. A study of factorial structure of the Persian version of the multidimensional peer-victimization scale in primary schools students in Semnan city. Koomesh. 2016; 17(3):660-8. https://www.cabdirect.org/cabdirect/abstract/20163114195

[46] Goss K, Gilbert P, Allan S. An exploration of shame measuresI: The other as Shamer scale. Personality and Individual Differences. 1994; 17(5):713-7. [DOI:10.1016/0191-8869(94)90149-X]

[47] Foroughi A, Khanjani S, Kazemini M, Tayeri F. [Factor structure and psychometric properties of Iranian version of External Shame Scale (Persian)]. Shenakht Journal of Psychology and Psychiatry. 2015; 2(2):49-58. https://www.sid.ir/en/journal/ViewPaper.aspx?ID=568397

[48] Raes F, Pommier E, Neff KD, Van Gucht D. Construction and factorial validation of a short form of the Self-Compassion Scale. Clinical Psychology \& Psychotherapy. 2011; 18(3):250-5. [DOI:10.1002/cpp.702] [PMID]

[49] Khanjani S, Foroughi AA, Sadghi K, Bahrainian SA. [Psychometric properties of Iranian version of self-compassion scale (short form) (Persian)]. Pajoohandeh Journal. 2016; 21(5):282-9. http:// pajoohande.sbmu.ac.ir/article-1-2292-en.html

[50] Angold A, Costello E. Mood and feelings questionnaire [MFQ]. Durham: Developmental Epidemiology Program, Duke University; 1987. [DOI:10.1037/t15197-000]

[51] Neshatdoust HT, Nouri N, Molari H, Kalantari M, Mehrabi HA. [Standardization of Mood and Feeling Questionnaire (Persian)]. Journal of Psychology. 2006; 9(4):334-50. https:/ / www.sid. ir/en/Journal/ViewPaper.aspx?ID=50731

[52] Miles J, Shevlin M. Applying regression and correlation: A guide for students and researchers. Thousand Oaks, California: Sage; 2001.

[53] Kline RB. Principles and practice of structural equation modeling. New York: Guilford; 2001.

[54] Preacher KJ, Hayes AF. Asymptotic and resampling strategies for assessing and comparing indirect effects in multiple mediator models. Behavior Research Methods. 2008; 40(3):879-91. [DOI:10.3758/BRM.40.3.879] [PMID]

[55] Roth-Cline M, Nelson RM. Parental permission and child assent in research on children. The Yale Journal of Biology and Medicine. 2013; 86(3):291-301. [PMCID] [PMID] 